\title{
Glaciarismo en la divisoria cantábrica: \\ La evolución del glaciarismo Cuaternario en la Sierra de Cebolleda (León) ${ }^{1}$
}

\section{Glaciations in the Cantabrian Divide: The Quaternary glacial evolution in the Sierra de Cebolleda (León)}

\author{
Enrique Serrano Cañadas*, Manuel Gómez Lende*, \\ Juan José González Trueba**, María González García***
}

\section{INTRODUCCIÓN}

El glaciarismo pleistoceno de la Cordillera Cantábrica es bien conocido en cuanto a su distribución y extensión máxima. Desde que a finales del siglo xIX Penck iniciara su estudio y en el s. xx Obermaier profundizara en los Picos de Europa como aportación al glaciarismo de la Península Ibérica, se han estudiado la mayoría de los complejos morrénicos, circos y macizos de la cordillera. Las fases establecidas inicialmente, basadas en el poliglaciarismo, fueron matizadas por las características locales de las montañas cantábricas (ver Frochoso y Castañon, 1998; Redondo et al. 2002) y entre los años 60 y 90, los geógrafos españoles completaron el conocimiento geomorfológico del máximo glaciar en estas montañas. La fase de máxima extensión, registrada con nitidez en el relieve, es la mejor conocida (Castañón y Frochoso, 1992; Frochoso y

${ }^{1}$ Este trabajo ha sido realizado en el marco de los proyectos OAPN-053/2010 del Organismo Autónomo Parques Nacionales y CGL-2010-19729 del Plan Nacional de Investigaciones Científicas y Tecnología, así como con la ayuda del Parque Nacional Picos de Europa.

* Departamento de Geografía. Universidad de Valladolid.

** CIESE-Comillas. Universidad de Cantabria.

*** PANGEA, Universidad de Valladolid. 
Castañón, 1998; Redondo et al. 2002; Pérez Alberti et al. 2004; Jalut et al. 2010; Moreno et al. 2010; Serrano et al., 2013; Jiménez et al. 2012), así como la última pulsación, la Pequeña Edad del Hielo (González Trueba, 2006, 2007; González Trueba et al. 2008), ya de época histórica. Trabajos posteriores (Serrano y González Trueba, 2002; González Gutiérrez, 2002; González Trueba, 2006; Santos, 2011; Pellitero, 2012, 2013), atienden a la reconstrucción de la evolución glaciar durante el Pleistoceno y el Holoceno y sitúan la máxima extensión del glaciarismo anterior al Último Máximo Glaciar ${ }^{2}$ europeo, con una sucesión de fases de retroceso sin atribución cronológica precisa. Es una hipótesis de trabajo que necesita una continuidad en la labor de futuro para el conocimiento más preciso de los cambios ambientales acaecidos en el norte de la Península Ibérica durante el Cuaternario. Todavía existe un gran vacío en relación con posibles glaciaciones, avances y retrocesos ocurridos entre la máxima expansión y el glaciarismo histórico, comprendiendo al menos los últimos 40.000 años.

En la Sierra de Cebolleda, Alonso et al. (1982) establecieron la existencia de morfologías glaciares, circos y morrenas, realizando una primera descripción de las mismas, y Castañón (1989) delimita la máxima extensión de los glaciares de este conjunto, cartografiando sus frentes. Alonso Herrero (1987, 2002) estudia las características morfológicas generales, señala la inexistencia de lenguas hacia el sur y la presencia de glaciares rocosos, y cartografía los depósitos y formas glaciares más importantes de los circos en la zona de estudio. Pellitero et al. (2011) han estudiado los glaciares rocosos de la sierra, estableciendo su tipología y su relación con una única fase de génesis de glaciares rocosos, asociada a periodos cataglaciares y ambientes paraglaciares de las últimas fases de deglaciación y periodos fríos del Pleistoceno.

La importancia de acotar la extensión superficial y cronológica de cada fase glaciar incide de modo directo en el conocimiento de la evolución ambiental y climática, en el establecimiento y caracterización de las fases frías o templadas y su importancia geomorfológica. Pero también las relaciones con una ocupación humana intensa del territorio en este periodo final del Pleistoceno, cuando las incipientes sociedades humanas eran dependientes del medio y se producen migraciones del hombre moderno o la extinción del neandertal. Por ello, es hoy de sumo interés conocer en qué momento avanzaron los glaciares, qué espacios ocuparon y cómo se comportaron en relación con los cambios ambientales y el poblamiento humano de la Cordillera Cantá-

\footnotetext{
2 Último Máximo Glaciar, LGM en adelante.
} 
brica en un ambiente peninsular muy diferente del mediterráneo dominante. Para ello, el estudio y conocimiento de cada macizo, incluida la Sierra de Cebolleda, pequeño macizo situado entre los dos más altos de la Cordillera Cantábrica (Picos de Europa al norte y Peña Prieta al sur), de su singularidad y evolución glaciar cobra importancia para la interpretación del conjunto del glaciarismo de la Cordillera Cantábrica.

En este trabajo se estudia la evolución glaciar en la Sierra de Cebolleda, y las diferentes fases de avance o equilibrio que han dejado huellas geomorfológicas. Estas fases se sitúan en el tiempo y se asocian a condiciones ambientales diferenciadas en función de sus características. El objetivo es conocer con más precisión la evolución y extensión del glaciarismo en cada fase y los caracteres principales (altitud de los frentes, de la Altitud de la línea de Equilibrio Glaciar $^{3}$, tipo de glaciares) para establecer correlaciones lo más precisas posibles con los restantes macizos cantábricos.

\section{Metodología}

La cartografía geomorfológica es una herramienta básica para el conocimiento de las formas de relieve que permite la representación exacta de las formas y establecer las relaciones espaciales entre los distintos elementos, de modo que constituye una herramienta de análisis. Pero también es un documento básico para el conocimiento y la gestión de los territorios de montaña. El mapa geomorfológico permite caracterizar cada sistema de formas y reconstruir detalladamente los glaciares Pleistocenos de cada fase glaciar. El análisis morfoestratigráfico, partiendo de una sólida base cartográfica, es una técnica adecuada para la reconstrucción de la evolución y el establecimiento de cronologías relativas mediante la localización temporal de cada glaciar y glaciar rocoso utilizados como indicadores de fases glaciares en el primer caso (Lukas, 2006; Huhges, 2010) o fases frías para los segundos (Barsch, 1996; Haeberli et al. 2006). Las correlaciones glaciares entre cada valle incluyen datos procedentes del mapa (orientación, altitud de los complejos morrénicos, longitud y tipo de glaciar) y de la estimación de la ELA. Cinco glaciares rocosos han sido inventariados incluyendo datos de altitud, longitud, anchura, orientación, litología y periodización.

\footnotetext{
${ }^{3}$ Altitud de la línea de Equilibrio Glaciar, ELA en adelante.
} 
El mapa geomorfológico de la Sierra de Cebolleda sigue las directrices utilizadas para la cartografía E1/25.000 de los Picos de Europa (González Trueba, 2007; González Trueba y Serrano, 2010; González Trueba et al. 2011, 2012), completando un sector de la porción meridional del Parque. Ésta limita con la Montaña Palentina, donde también se dispone de una cartografía geomorfológica de detalle (Pellitero, 2009, 2012). El mapa utiliza la base topográfica del Instituto Geográfico Nacional (porción centro-oriental del Mapa Topográfico Nacional de España, E. 1/25.000. Hoja n ${ }^{\circ} 80-$ II y 80-IV. Burón. 2002, I.G.N.) y la base cartográfica del Parque Nacional de los Picos de Europa. Basado en los sistemas cartográficos francés, RCP 77 y del Institute de Geographie Université de Lausanne-IGUL, con adaptaciones gráficas y de contenidos realizadas en función de los sistemas aceptados, se realiza una clasificación morfológica y genética de las formas de relieve, con un total de 34 formas y depósitos representados en la zona de estudio, destacando las formas glaciares (circos glaciares, cubetas de sobreexcavación, umbrales, artesas, formas menores, complejos morrénicos, depósitos de obturación lateral y depósitos de colmatación). La elaboración cartográfica implica el análisis e interpretación de las formas y el entendimiento global de la interacción del relieve con el resto de hechos naturales y humanos en el tiempo, de modo que el relieve de Cebolleda se comprenda como parte esencial del sistema natural.

La ELA es la altitud a la que el balance de masa es igual a cero y está condicionada por las relaciones entre precipitaciones y temperaturas, por lo que es un parámetro muy útil para la caracterización ambiental de los ambientes glaciares (Porter, 1975; Seltzer, 1994; Benn y Ballantyne, 2005) pues permite una aproximación a las condiciones ambientales y las variaciones regionales del glaciarismo Pleistoceno. Los factores que influyen en la ELA de cada glaciar son el albedo, los derrubios supraglaciares, la topografía, la orientación, la sobreacumulación de nieve por aludes y el viento, la geometría del glaciar, la hipsometría, las nevadas de invierno y verano y las temperaturas (Ohmura et al. 1992; Seltzer, 1994; Nesje, 2006; Bakke y Nesje, 2011), de modo que las relaciones entre el clima y la ELA son complejas y cada glaciar se individualiza respecto al clima general. El valor medio de paleoELA de un macizo indica las condiciones teóricas glaciares de equilibrio y permite comparaciones regionales. En la Sierra de Cebolleda se han calculado 25 ELA pertenecientes a cuatro fases glaciares diferenciadas. Para la reconstrucción de las paleoELAs, la ELA de glaciares Cuaternarios, se ha aplicado el método Accumulation Area Ratio ${ }^{4}$,

\footnotetext{
${ }^{4}$ Accumulation Area Ratio, AAR en adelante.
} 
basado en la relación entre el balance de masa y el porcentaje del área de acumulación respecto a su área total, asumiendo un porcentaje del AAR de $0,6 \pm 0,05$ o $60 \% \pm 5 \%$, característico de los glaciares de valle en latitudes medias (Hawkins, 1985; Kuhle, 1988; Dahl y Nesje, 1992). La ELA se ha calculado a partir de una hoja de cálculo con tecnología SIG considerando la extensión glaciar y la topografía por rangos altitudinales. Este método ha sido ampliamente utilizado y entre los existentes, todos ellos con deficiencias derivadas de la reconstrucción glaciar, se considera el más eficaz para estimar las líneas de equilibrio glaciar de antiguos glaciares cuando se dispone de cartografía adecuada y la posibilidad de una reconstrucción precisa (Bakke y Nesje, 2011).

La cronología relativa de las formas (morrenas, conos proglaciares, derrubios de ladera, glaciares rocosos), permite reconstruir la glaciaciones cuaternarias y su evolución (Lukas, 2006; Hughes, 2010) y atribuirles unas condiciones ambientales precisas. Por ello el análisis morfoestratigráfico es el paso obligado previo a la obtención de dataciones absolutas para una correcta interpretación cronológica de las formas. En el macizo se ha analizado la ubicación de los complejos morrénicos escalonados, en función de su altitud, posición en los valles y altitud de la ELA, y a partir de la periodización de avances o equilibrios glaciares en cada valle, se ha establecido las principales fases morfogenéticas de acumulación glaciar. La correlación morfoestratigráfica entre los valles se ha hecho incluyendo datos sobre la orientación, la altura de los frentes, la longitud de los glaciares y la altitud de la línea de equilibrio glaciar (ELA). A partir de los elementos analizados se propone una reconstrucción ambiental de cada una las fases glaciares (Ballantyne, 2002; Benn y Ballantyne, 2005) que permite diferenciar las condiciones ambientales que posibilitaron el desarrollo y permanencia de los glaciares de montaña en la Sierra de Cebolleda.

\section{LA ZONA DE ESTUDIO}

La Sierra de Cebolleda (436'1" N-45' $48^{\prime \prime} W$ ) se localiza en la divisoria cantábrica, separando las cuencas del Cares-Deva (Cantábrica) y Esla (Duero) al sur del macizo Occidental de los Picos de Europa (Fig.1). Forma un cordal de dirección E-W y $10 \mathrm{~km}$ de longitud entre el puerto de Panderrueda y el río Tuerto, al oeste, y el Puerto de Pandetrave al oeste. El cordal se emplaza a altitudes mayores de $1700 \mathrm{~m}$ y en él destacan las cumbres de Gabanceda (2042), Gildar (2074) y Cebolleda (2054). Sobre este cordal se abren, al norte y al sur, valles de dirección meridiana que estuvieron glaciados durante el Pleistoceno. 
Su posición al sur de las montañas más altas de la Cordillera Cantábrica la sitúa al cobijo de los frentes del $\mathrm{N}$ y NW, de modo que una gran parte de la humedad procedente del mar no alcanza estas cumbres de altitudes moderadas donde en la actualidad se registran unas precipitaciones mayores de 1200 $\mathrm{mm} \mathrm{a}^{-1}$ (Allende, 2008) que contrastan con los más de $2500 \mathrm{~mm} \mathrm{a}^{-1}$ de precipitación registrada en los Picos de Europa, a sólo $10 \mathrm{~km}$ al norte, o los 2700 $\mathrm{mm} \mathrm{a}^{-1}$ registrados en Peña Prieta, a sólolo km al sur. El régimen de precipitaciones en este sector de la Cordillera Cantábrica deriva de los accesos de ciclones profundos del $\mathrm{N}$ y NW, determinados por la organización orográfica y con fuertes contrastes por sombra orográfica (Allende, 2008). Se trata, pues, de

FIGURA 1

LOCALIZACIÓN DEL ÁREA DE ESTUDIO Y SUPERFICIE DEL PARQUE NACIONAL PICOS DE EUROPA

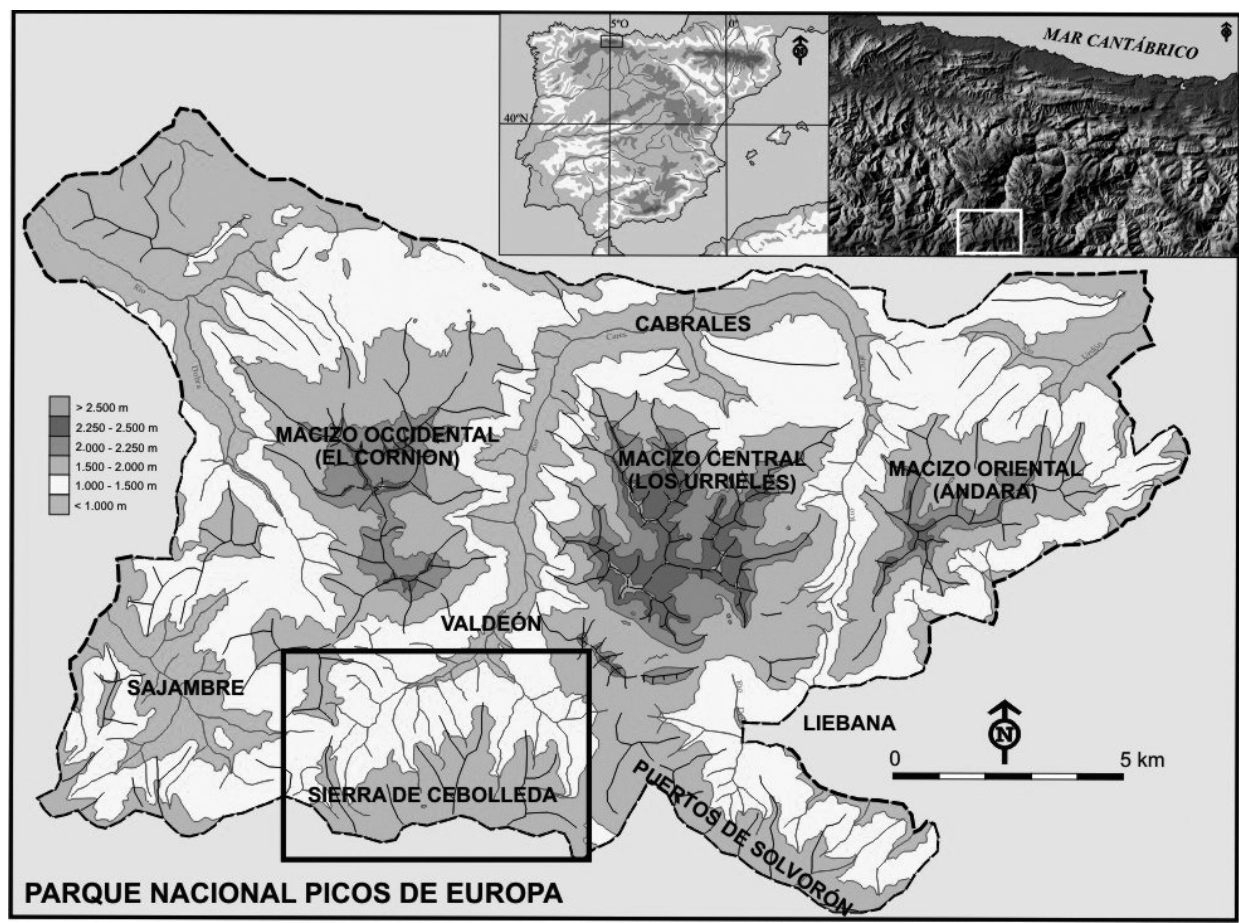

Fuente: elaboración propia.

Estudios Geográficos, Vol. LXXVI, 278, pp. 335-367, enero-junio 2015

ISSN: 0014-1496, eISSN: 1988-8546, doi: 10.3989/estgeogr.201512 
una zona de la divisoria que no constituye el primer resalte frente al mar, por lo que las condiciones oceánicas de estas montañas son más moderadas que en los macizos más septentrionales.

El núcleo principal de la Sierra de Cebolleda se levanta en la Unidad geológica Gildar-Montó perteneciente a la Zona Cantábrica, en la parte NW de la región del Pisuerga-Carrión, y en un contexto de complicada tectónica epidérmica (Fig.2). Predominan mantos, pliegues y líneas de fracturas directas e inversas (Julivert 1971; Brouwer, 1964; Frankenfeld, 1983; Marquínez y Marcos, 1984), y se han reconocido cinco escamas de cabalgamiento y plegamientos menores con ejes NE-SW (sinclinales del Cordal y Gildar, anticlinal del Cable, Figs. 2 y 3). La Unidad Tildar-Montó constituye un manto alóctono levantado y desplazado con respecto a sus raíces más meridionales (Frankenfeld, 1983; Marquínez y Marcos, 1984; Rodríguez Fernández y Heredia, 1988; Heredia y Rodríguez Fernández, 1990). A su vez, este manto se encaja entre materiales más recientes del Carbonífero superior, mantos autóctonos de menor complejidad tectónica (Fig.3) con frentes de cabalgamiento de vergencia SE, fracturados y desnivelados por fallas tardías (ENE-WSW, NW-SE y N-S). Estas afectaron a la falla directa WNW-ESE de Cable, provocando el hundimiento de su labio septentrional (Marquínez y Marcos, 1984). La orogenia Alpina elevó este conjunto y configuró una morfoestructura monoclinal, con frentes orientados al SE y dorsos al NW, con klippes en la vertiente septentrional constituidas por calizas carboníferas de montaña, calizas griotte, calizas de la Fm. Alba y la Fm. Vidrieros. En el frente meridional afloran materiales silíceos de menor resistencia que configuran cabeceras homogéneas, con resaltes a favor de afloramientos conglomeráticos y brechas calcáreas que constituyen las cumbres de Gabanceda y Picos de la Silla (Figs. 2 y 3 ).

Esta estructura geológica confiere a la sierra una amplia diversidad litológica. En las laderas septentrionales y los fondos del valle de Valdeón afloran areniscas y cuarcitas Devónicas (fm. Murcia), y ya en el manto de Valdeón, al norte, areniscas, lutitas y conglomerados de edad Estefaniense de los grupos Valdeón y Pontón. Al sur, afloran las lutitas, areniscas y conglomerados de la fm. Pandetrave y el Grupo Maraña, abriéndose en él los valles ortoclinales meridionales, y en los materiales más competentes las cumbres de la Sierra de Gabanceda (Peña de Gabanceda, Peña La Silla, Alto de Vallosera).

Los materiales y la estructura descritos configuran una estructura monoclinal, topográficamente disimétrica y de dirección E-W que ha condicionado el glaciarismo cuaternario. Presenta frentes al sur, con fuerte pendientes, donde 
FIGURA 2

BLOQUE DIAGRAMA Y MORFOESTRUCTURAS DE LA SIERRA DE CEBOLLEDA Y SU ENTORNO

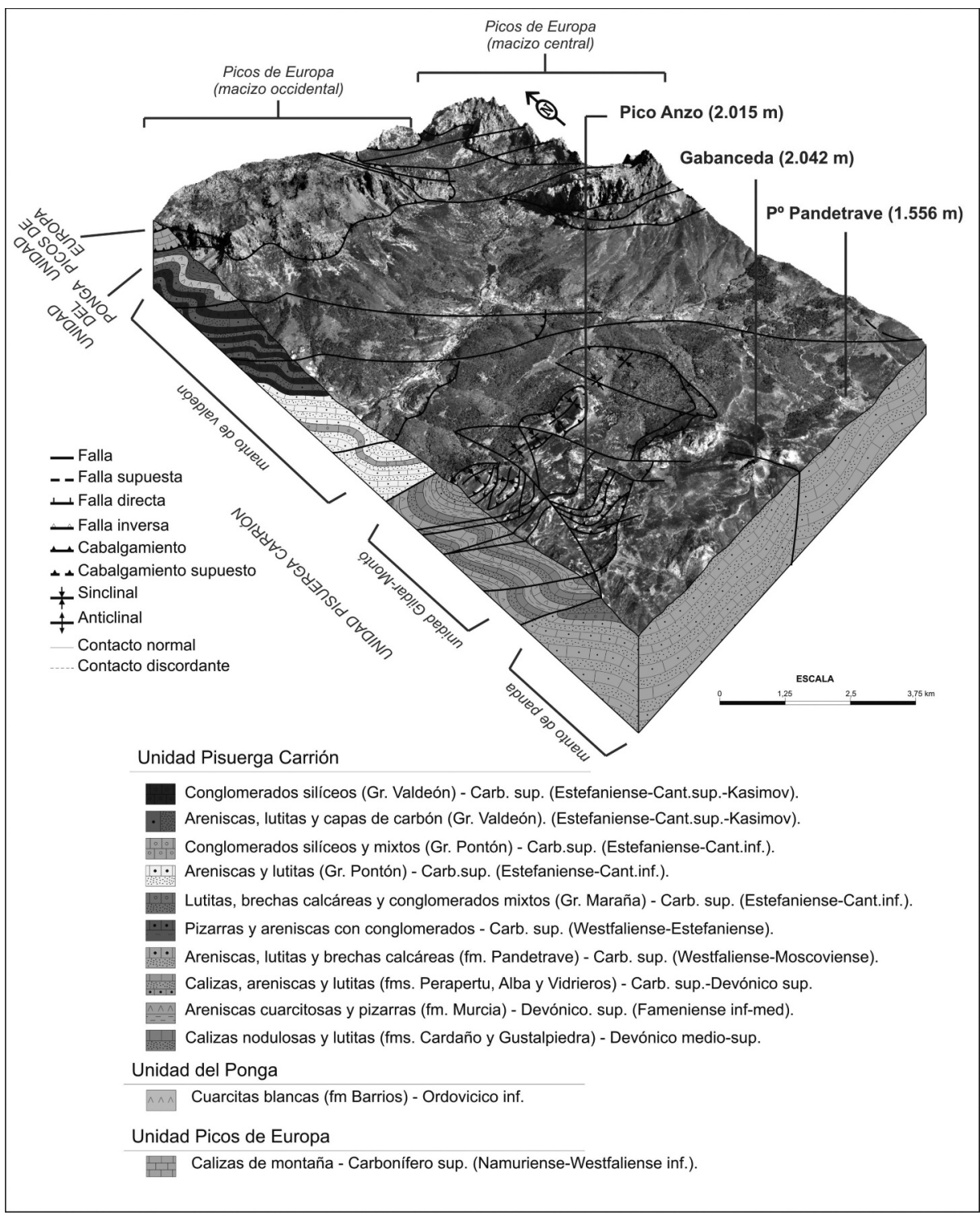

Fuente: elaboración propia. Litología y tectónica adaptadas de Marquínez y Marcos (1984).

Estudios Geográficos, Vol. LXXVI, 278, pp. 335-367, enero-junio 2015

ISSN: 0014-1496, eISSN: 1988-8546, doi: 10.3989/estgeogr.201512 
los glaciares fueron de pequeña extensión, y amplios dorsos fallados y cabalgados al norte, propicios para la erosión diferencial y el desarrollo de glaciares de lengua. En la línea divisoria, la estructura propició la diversidad litológica y morfológica de las cumbres y es la responsable de la pronunciada disimetría glaciar N-S de la Sierra de Cebollera.

\section{FIGURA 3}

\section{DETALLE DE LAS ESCAMAS DE LA UNIDAD DE GILDAR-MONTÓ. ADAPTADO DE MARQUÍNEZ Y MARCOS (1984)}

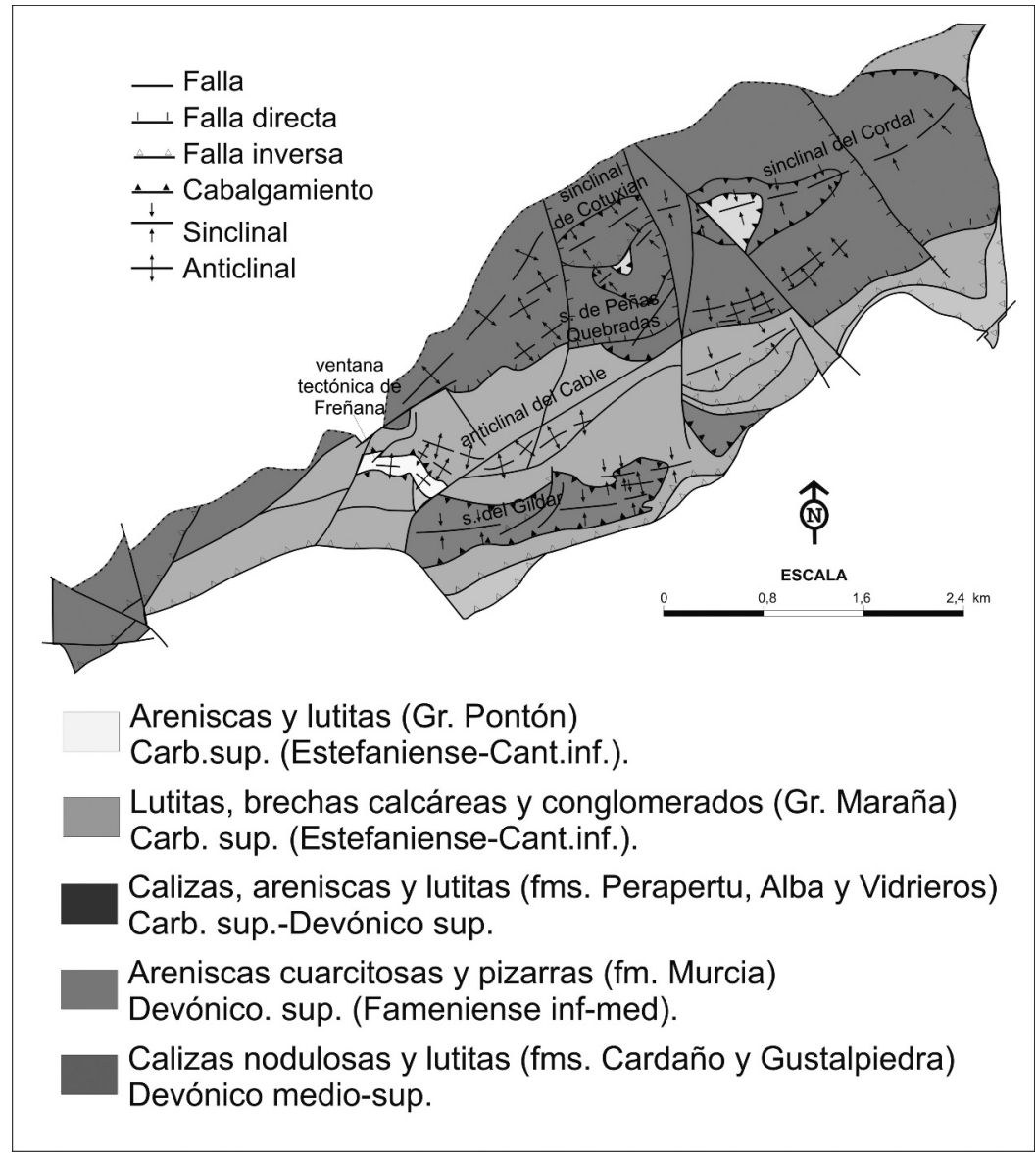

Fuente: elaboración propia. 
MORFOLOGÍA GLACIAR, LÍNEAS DE EQUILIBRIO GLACIAR Y MORFOESTRATIGRAFÍA

Sobre el cordal principal, al norte y al sur, y los secundarios que se alargan hacia el norte, se abren 12 valles de dirección meridiana que durante el Pleistoceno estuvieron ocupados por glaciares (Fig.4), orientados preferentemente al norte y al sur (Fig.5). Los glaciares remodelaron los valles y generaron formas en artesa características, amplios circos en las cabeceras y complejos morrénicos en los frentes y valles. Para este trabajo se han estudiado y reconstruido 11 circos y sus respectivas lenguas (tabla 1 ):

- Glaciar de Freñana: valle glaciar con el circo orientado al norte y la lengua al NW que drenaba sus aguas hacia el Esla. Formó un glaciar simple, que partiendo del circo, de dimensiones y altitud moderada (pico Freñana, $1866 \mathrm{~m}$ ) organizó una lengua de 2,4 km de longitud con su frente a 1328 m s.n.m. y la ELA estimada a $1500 \pm 22$ m (Fig. 6 A). Esta lengua presenta un complejo morrénico frontal bien conservado y una morrena interna localizada a $1365 \mathrm{~m}$ s.n.m. configurando un glaciar de 2,1 kms, con la ELA estimada a $1538 \pm 6 \mathrm{~m}$, sólo 38 metros más elevada que la anterior. Valle arriba se localizan un arco morrénico frontal que ha permitido reconstruir un glaciar de $567 \mathrm{~m}$ de longitud cuyo frente se sitúo a 1590 m s.n.m. y la ELA a $1688 \pm 6 m$, significativamente más alta que en las anteriores. Al pie de las paredes se localiza un nuevo complejo morrénico desdoblado, a $1722 \mathrm{~m}$ s.n.m y la ELA estimada a $1722 \pm 4 \mathrm{~m}$, orientado al norte y junto a un pequeño glaciar rocoso.

- Glaciar de Cebolleda: valle glaciar curvo, que a partir del circo, orientado al norte, toma dirección NW para arquearse hacia el NE en su porción final. Durante el máximo glaciar formó una lengua de 4,3 km de longitud capaz de depositar un complejo morrénico frontal que no alcanzó el fondo del valle del Cares (Valdeón). Este complejo está compuesto por dos morrenas frontolaterales que alcanzan los $1230 \mathrm{~m}$ s.n.m. y permite reconstruir un glaciar de 4,3 kms de longitud. La ELA se sitúa a 1540 49 $\mathrm{m}$. Al interior, se han conservado, $400 \mathrm{~m}$ más atrás, una morrena frontolateral que señala la posición del frente a 1340 m s.n.m, con la ELA situada a $1619 \pm 26 \mathrm{~m}$, conformando un glaciar de $3,8 \mathrm{kms}$ de longitud. Valle arriba, a 1540 m s.n.m., se encuentra otro arco morrénico frontolateral, junto a una morrena frontal de un valle lateral, posiblemente coetánea, que señala una fase glaciar definida por un glaciar de lengua, de 1,8 $\mathrm{km}$ de longitud, cuya porción principal de hielos se encuentra en el circo, donde se situaría la ELA, a 1739 $15 \mathrm{~m}$. Otras morrenas se alojan en el interior del circo (Fig.6B), señalando fluctuaciones menores adscritas 


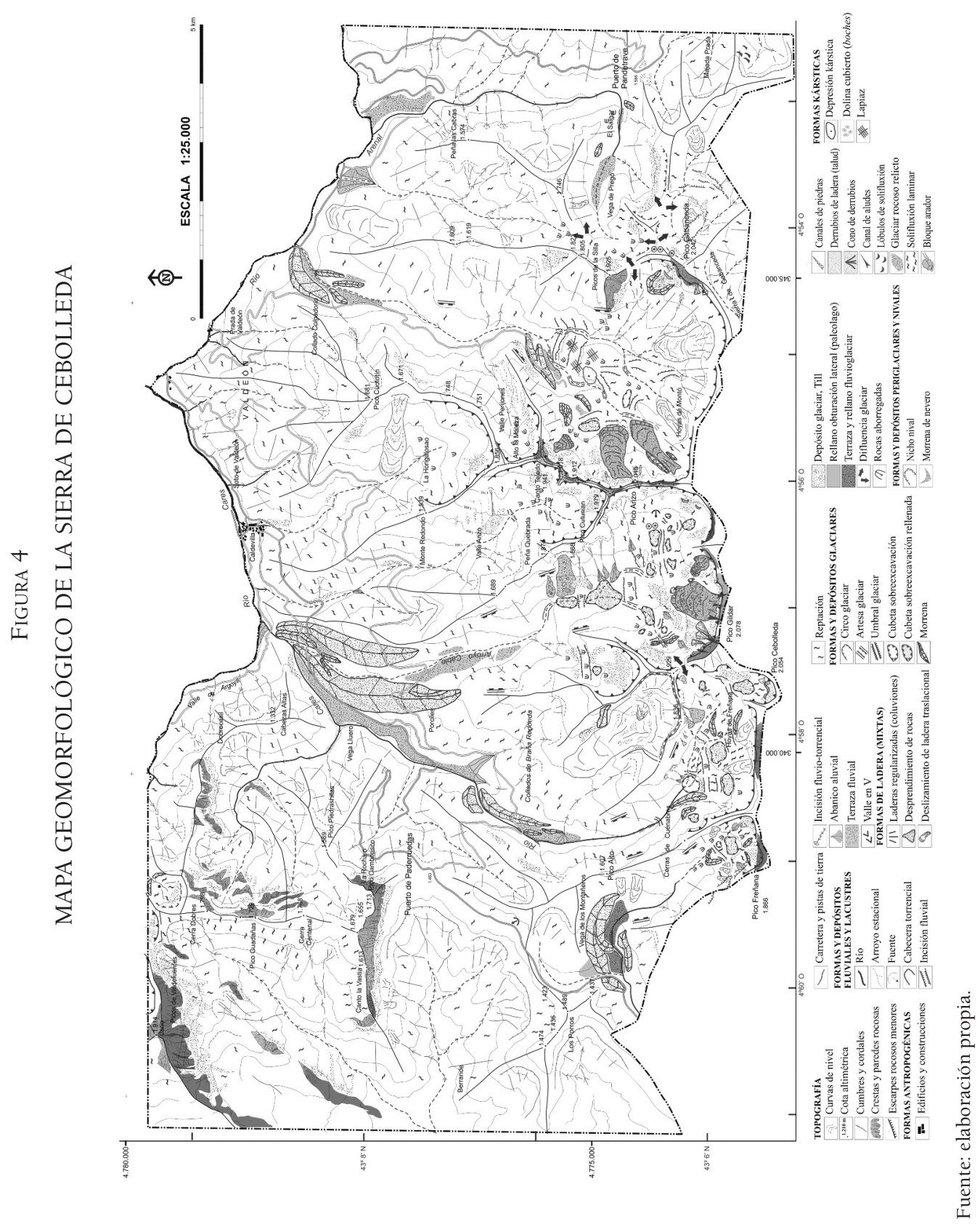

Estudios Geográficos, Vol. LXXVI, 278, pp. 335-367, enero-junio 2015 ISSN: 0014-1496, eISSN: 1988-8546, doi: 10.3989/estgeogr.201512 
al retroceso glaciar, sin posibilitar la reconstrucción de glaciares. Finalmente, el circo colgado en la cara norte de Cebolleda alberga una morrena frontal que señala un periodo final, con el glaciar por encima de los $1860 \mathrm{~m}$ s.n.m., una longitud de $420 \mathrm{~m}$ y la ELA a $1908 \pm 5 \mathrm{~m}$ de altitud. Todavía existen huellas de frío en su interior, con morrenas de nevero, hoy semifuncionales, alojadas al pie de las paredes de Cebolleda.

- Glaciar de Gildar: es el glaciar más extenso de la sierra de Cebolleda, con más de $5 \mathrm{~km}$ de longitud en su máxima expansión. Fue un glaciar generado en un amplio circo compuesto que alimentó una artesa glaciar de 3 $\mathrm{km}$, con el frente a 1010 m s.n.m. El complejo morrénico frontal alcanzó el fondo del valle, obturando el río Cares y emplazando dos grandes arcos principales y sucesivos arcos menores, hasta tres bien señalados. La ELA se sitúa a $1488 \pm 55 \mathrm{~m}$. Este glaciar muestra un retroceso entrecor-

\section{FIGURA 5}

\section{GLACIARES DE LA SIERRA DE CEBOLLEDA EN EL MÁXIMO GLACIAR CUATERNARIO}

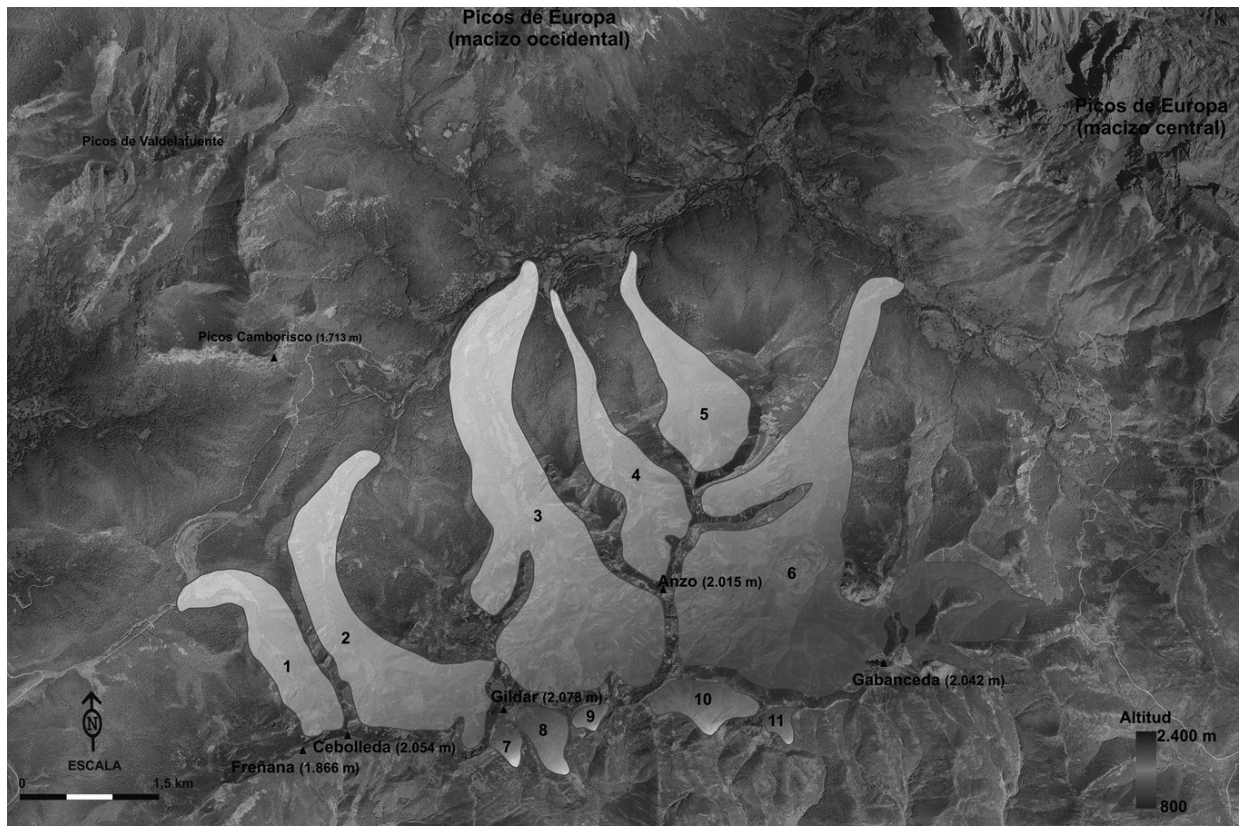

(1) gl. Freñana; (2) gl. Cebolleda; (3) gl. Gildar; (4) gl. Anzo; (5) gl. Monte Redondo; (6) gl. Gabanceda; (7) gl. Gildar Sur; (8) gl. Cosgaya; (9) gl. Las Verdes de Cable; (10) gl. Orza; (11) gl. La Requejada. Fuente: elaboración propia. 
tado, con la morrena más interna del complejo 1500 m más arriba, señalando un glaciar menor, con un frente en trono a 1240 m s.n.m y la ELA a $1606 \pm 30 \mathrm{~m}$. Valle arriba no existen complejos bien conservados que permitan reconstruir fases glaciares de retroceso. La amplitud del circo, poco profundo y ancho, pudo no propiciar estadios de equilibrio capaces de generar complejos morrénicos, o los procesos postglaciares han destruido las formas, pero muestra una clara discordancia con la evolución de los valles que se localizan al este y oeste. Ya en los circos las morrenas señalan la existencia de un glaciar de reducido tamaño, con 260 $\mathrm{m}$ de longitud, por encima de $1450 \mathrm{~m}$ s.n.m., orientado al NE, y al norte sendos glaciares rocosos de grandes dimensiones (Fig. 6C).

- Glaciar de Anzo: pequeño circo de altitud moderada (pico Anzo, 1950 $\mathrm{m}$ ) que alimentaba una lengua de fuerte pendiente y alcanzaba el fondo de valle, con el frente a 1040 m s.n.m. Los depósitos morrénicos y bloques erráticos denotan su extensión y dimensiones, favorecidas por la orientación $\mathrm{N}$ y la fuerte pendiente, con $900 \mathrm{~m}$ de desnivel en $3 \mathrm{~km}$.

- Glaciar de Monte Redondo: pequeño glaciar de 2,6 km de longitud, con un circo y lengua orientados al norte, de fuerte pendiente y altitud moderada (Monte Redondo, $1856 \mathrm{~m}$ ), que alcanzó las proximidades de Caldevilla, como apuntan los depósitos morrénicos y bloques erráticos de la ladera este del arroyo de Arriba en las proximidades de la población.

- Glaciar de Gabanceda: se trata del segundo glaciar por dimensiones de la sierra, que alcanzaba los 1115 m s.n.m. en el río Arenal. Alimentado por un amplio circo glaciar, formó una lengua de $5,2 \mathrm{~km}$ de longitud que modeló un complejo morrénico frontal formado por una morrena externa y tres arcos internos con sucesivos rellanos de obturación. Esta lengua estaba alimentada por un segundo circo, Valle Pericones, que favorecía la alimentación y desarrollo del glaciar, cuya ELA se localizaba a $1600 \pm 27$ m. A la salida del circo, a 1425 m s.n.m. se localiza una morrena frontolateral desdoblada, que señala la existencia de un glaciar de $2,25 \mathrm{~km}$ de longitud caracterizado por una lengua de reducidas dimensiones y un amplio circo situado por encima de los $1650 \mathrm{~m}$ s.n.m. (Fig. 6E), donde se sitúa la ELA $\left(1671_{ \pm} 10 \mathrm{~m}\right)$. Fue el glaciar más grande de los alojados en los circos, y denota la persistencia del hielo a favor de circos amplios y profundos. Por encima se registran en las laderas del circo sendos complejos morrénicos frontales, por encima ya de los 1590 m s.n.m. al ENE y 1750 m s.n.m. al NW (Fig. 6D). Estas formas señalan un periodo marginal, con glaciares muy pequeños ocupando ámbitos topoclimáticos favorables acompañados de glaciares rocosos (Fig. 7). 


\section{FIGURA 6}

\section{MORFOLOGÍA GLACIAR EN LA SIERRA DE CEBOLLEDA.}
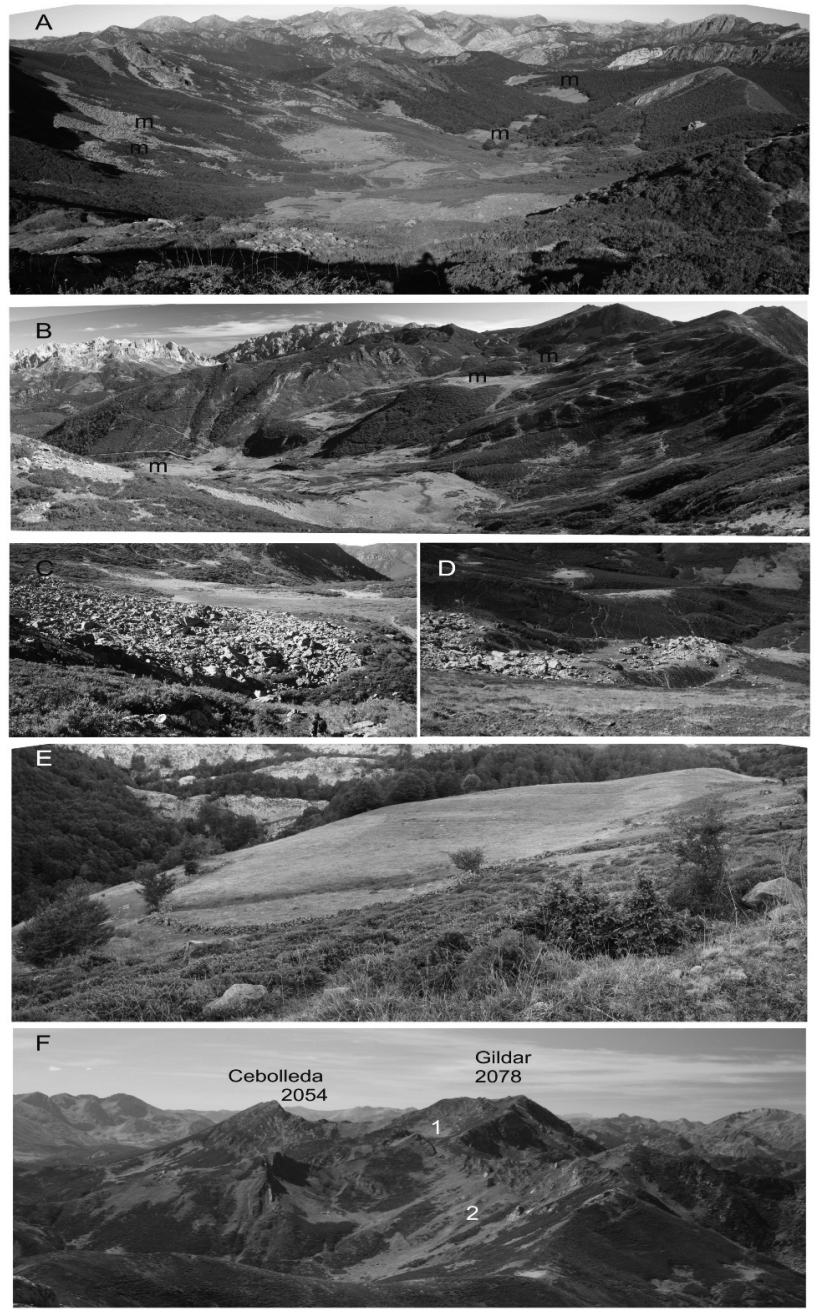

A, circo y artesa glaciar de Freñana. Se aprecian las morrenas (m) de las fases I, III y IV. B, circo glaciar de Cebolleda. m, morrenas. C, Frente del glaciar rocoso de los Verdes de Cable, a 1820 m s.n.m, en el circo de Gildar. D, morrenas frontales de la Fase IV en la cara norte del pico Gabanceda. E, Morrena frontal interna de Gabanceda, perteneciente a la fase III y situada a 1240 m s.n.m. F, circos glaciares en la vertiente meridional. 1, circo de Cosgaya; 2, Circo de Los Verdes de Cable. D, Complejo morrénico frontal de Gabanceda (F-IV). E, Morrena frontolateral y bloques erráticos en el valle de Gabanceda, a 1425 m s.n.m. F, Cordal de la Sierra de Cebolleda y morfología glaciar de la vertiente sur, circos de Gildar (1) y Orza (2).

Fuente: elaboración propia.

Estudios Geográficos, Vol. LXXVI, 278, pp. 335-367, enero-junio 2015

ISSN: 0014-1496, eISSN: 1988-8546, doi: 10.3989/estgeogr.201512 
- Glaciar de Gildar Sur: al pie de las cumbres más elevadas de la sierra, en su vertiente sur, se localiza un circo glaciar cuyas morrenas se sitúan a $1761 \mathrm{~m}$ s.n.m. Es un pequeño circo glaciar de $460 \mathrm{~m}$ de longitud colgado en la ladera, con la ELA a $1868 \pm 12 \mathrm{~m}$, en el que no se aprecian formas morrénicas.

- Glaciar de Cosgaya: al sur del Pico Gildar se desarrolla el circo más grande de la vertiente sur, cuyas formas glaciares permiten reconstruir una lengua incipiente de $875 \mathrm{~m}$ de longitud que alcanzó los $1698 \mathrm{~m}$ s.n.m. (Fig. 6F).

- Glaciar de Los Verdes de Cable: pequeño circo glaciar que alojó un aparato de $285 \mathrm{~m}$ de longitud, cuyo frente se situaba a $1825 \mathrm{~m}$ s.n.m., desarrollado a favor de la cresta de Las Verdes de Cable.

- Glaciar de Orza: amplio circo glaciar de 1200 m de ancho, elaborado por un glaciar de circo acantonado en la cresta, de $740 \mathrm{~m}$ de longitud que alcanzó los 1670 m s.n.m. En su interior existen morrenas que denotan la existencia de un glaciar de $460 \mathrm{~m}$ de longitud, cuya ELA se situó $30 \mathrm{~m}$ más alta que el anterior.

- Glaciar de La Requejada: pequeño circo orientado al SE, al pie de una cumbre de 1912 m s.n.m., que albergó un glaciar de circo de 440 m de longitud. Se trata del último glaciar de la sierra en la vertiente sur, donde las cumbres descienden paulatinamente por debajo de los 1900 m s.n.m.

FIGURA 7

\section{GLACIARES ROCOSOS DEL CIRCO DE LAS HOYAS DE MONTÓ EN EL CIRCO DE GABANCEDA}

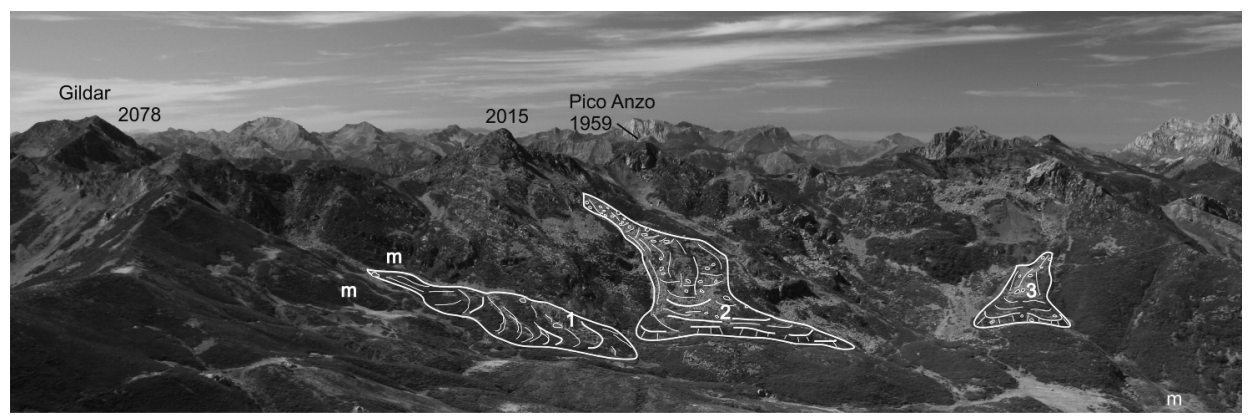

1, Glaciar rocoso de Montó 1. 2, Glaciar rocoso de Montó 2. 3, Glaciar rocoso de Montó 3. m, morrenas. Fuente: elaboración propia. 
TABLA 1

DATOS PALEOGLACIARES DE LA SIERRA DE CEBOLLEDA

\begin{tabular}{|c|c|c|c|c|c|c|c|c|}
\hline Glaciar & Fase & $\begin{array}{c}\text { Altitud } \\
\text { maxima } \\
(\mathrm{m})\end{array}$ & $\begin{array}{c}\text { Altitud } \\
\text { frente } \\
(\mathrm{m})\end{array}$ & Longitud & Or. & $\begin{array}{l}\text { Tipo de } \\
\text { glaciar }\end{array}$ & Cuenca & $\begin{array}{l}\text { ELA } \\
\text { (AAR) } \\
(\mathrm{m})\end{array}$ \\
\hline \multirow[t]{5}{*}{ Freñana } & I & 1866 & 1328 & 2440 & NW & Alpino & 1 & $1500 \pm 22$ \\
\hline & II & 1866 & 1365 & 2122 & NW & Alpino & 1 & $1538 \pm 6$ \\
\hline & III & 1866 & 1588 & 567 & NW & Alpino & 1 & $1688 \pm 6$ \\
\hline & IVa & 1866 & 1689 & 188 & NW & Circo & 1 & $1722 \pm 4$ \\
\hline & $\mathrm{IVb}$ & 1842 & 1742 & 130 & NE & Circo & 1 & $1768 \pm 2$ \\
\hline \multirow[t]{4}{*}{ Cebolleda } & I & 2078 & 1228 & 4314 & NW & Alpino & 1 & $1540 \pm 49$ \\
\hline & II & 2078 & 1338 & 3864 & NW & Alpino & 1 & $1619 \pm 26$ \\
\hline & III & 2078 & 1538 & 1868 & NW & Alpino & 1 & $1739 \pm 15$ \\
\hline & IV & 2054 & 1859 & 421 & $\mathrm{~N}$ & Circo & 1 & $1908 \pm 5$ \\
\hline \multirow[t]{3}{*}{ Gildar } & I & 2078 & 1009 & 5033 & NW & Alpino & 1 & $1488 \pm 55$ \\
\hline & II & 2078 & 1240 & 3596 & NW & Alpino & 1 & $1606 \pm 30$ \\
\hline & IV & 1929 & 1748 & 260 & $\mathrm{NE}$ & Circo & 1 & $1821 \pm 5$ \\
\hline Anzo & I & 1979 & 1042 & 3513 & NW & Alpino & 1 & $1512 \pm 35$ \\
\hline Monte Redondo & I & 1856 & 1000 & 2684 & NW & Alpino & 1 & $1375 \pm 35$ \\
\hline \multirow[t]{5}{*}{ Gabanceda } & I & 2042 & 1115 & 5205 & $\mathrm{NE}$ & Alpino & 1 & $1600 \pm 27$ \\
\hline & II & 2042 & 1168 & 4417 & $\mathrm{NE}$ & Alpino & 1 & $1615 \pm 24$ \\
\hline & III & 2042 & 1424 & 2254 & NE & Alpino & 1 & $1671 \pm 10$ \\
\hline & IVa & 1918 & 1590 & 830 & E & Circo & 1 & $1806 \pm 3$ \\
\hline & $\mathrm{IVb}$ & 2042 & 1749 & 339 & $\mathrm{NO}$ & Circo & 1 & $1688 \pm 10$ \\
\hline Gildar S. & I & 2078 & 1761 & 460 & SE & Circo & 2 & $1868 \pm 12$ \\
\hline Cosoya & I & 2078 & 1698 & 877 & SE & Circo & 2 & $1835 \pm 20$ \\
\hline Las Verdes de Cable & I & 1987 & 1825 & 285 & $S$ & Circo & 2 & $1883 \pm 3$ \\
\hline \multirow[t]{2}{*}{ Orza } & I & 1996 & 1670 & 738 & $S$ & Circo & 2 & $1782 \pm 9$ \\
\hline & II & 1996 & 1730 & 463 & $S$ & Circo & 2 & $1811 \pm 6$ \\
\hline La Requejada & I & 1912 & 1710 & 442 & SE & Circo & 2 & $1817 \pm 10$ \\
\hline
\end{tabular}

1, Vertiente cantábrica. 2, Cuenca del Duero, vertiente atlántica.

Fuente: elaboración propia.

Estudios Geográficos, Vol. LXXVI, 278, pp. 335-367, enero-junio 2015

ISSN: 0014-1496, eISSN: 1988-8546, doi: 10.3989/estgeogr.201512 
En conjunto la Sierra de Cebolleda presenta una fuerte disimetría norte sur, con glaciares de lengua bien desarrollados al norte y pequeños glaciares de circo al sur, que desaparecerían inmediatamente después del máximo o fueron tan pequeños que no han dejado huellas morfológicas de su presencia. La reconstrucción paleoglaciar de los once sistemas glaciares de la Sierra de Cebollera (tabla 1) muestra lenguas de más de $5 \mathrm{~km}$ en la vertiente norte, que alcanzaban los 1000 m s.n.m., y una altitud media de 1120 m s.n.m., en el fondo del valle de Valdeón. Fueron glaciares alpinos generados por encima de los 1600 m s.n.m., con lenguas capaces de remodelar los valles y generar complejos morrénicos bien conservados como los de Vega de los Montañeros, La Armadiella, LLadredo y las Carricias. La ELA si situaba en estos glaciares entre 1548 y 1600 metros de altitud, mostrando una gran homogeneidad en la vertiente septentrional. En sus frentes y el interior de los valles glaciados se suceden complejos morrénicos que señalan entre una y cuatro fluctuaciones glaciares con impronta geomorfológica, si bien los complejos más importantes, Freñana, Cebolleda y Gabanceda, denotan restos morfológicos (morrenas y depósitos) de cuatro fases morfogenéticas glaciares, dos próximas entre sí en cuatro de las grandes artesas. Esta proximidad está acompañada de un ascenso de $95 \mathrm{~m}$ en sus respectivas paleoMELAS. La sucesión de complejos morrénicos separados en la distancia y altitud e individualizados unos de otros, que se alojan en los valles glaciares y circos, permite establecer en cada valle y circo la sucesión glaciomorfológica, con diferentes fases de avance y equilibrio glaciar, que varían entre uno y cuatro para el conjunto:

- Glaciar de Freñana: El glaciar ha dejado restos morfológicos de cuatro pulsaciones principales, las dos primeras (F-I y F-II) muy próximas entre sí, con un glaciar de grandes dimensiones. Una nueva fase de retroceso señala un glaciar de lengua (F-III), y finalmente, alojado en altura, un pequeño glaciar junto a morrenas menores (F-IVa y IVb) que pasan a un glaciar rocoso en un ambiente de dominante periglaciar.

- Glaciar de Cebolleda: Este valle registra complejos morrénicos escalonados en las laderas pertenecientes a cuatro fases principales. Nuevamente se generan un máximo glaciar (F-I), en el fondo del valle, y un retroceso muy próximo (F-II) señalado por un complejo morrénico voluminoso, separado 450 metros de las anteriores. En el interior de la artesa glaciar se ubica un nuevo complejo morrénico que señala una fase de equilibrio interno (F-III) con pulsaciones menores. Finalmente, en los circos se lo- 
calizan morrenas que denotan un nuevo periodo de avance y equilibrio glaciar, una fase de altitud con glaciares muy reducidos (F-IV).

- Glaciar de Gildar: el glaciar más extenso de la sierra muestra tres fases glaciares bien señaladas. Dos complejos están próximos en la altitud y la ELA, situados en el fondo de valle pero separados a $1400 \mathrm{~m}$ de distancia (F-I y F-II), lo que señala dos posiciones diferenciadas del frente glaciar. En altitud, en el circo al pie de las paredes, un complejo morrénico orientado al este y glaciares rocosos al norte, señala una tercera y última fase glaciar, que por correlación con los valles contiguos a partir de la posición, tamaño, altitud, la ELA y por ser coetáneos de los glaciares rocosos, ha sido denominada como Fase IV.

- Glaciar de Gabanceda: El valle y circo de Gabanceda muestra huellas morfológicas de cuatro fases glaciares, dos propias de glaciares de grandes dimensiones (F-I y F-II), nuevamente próximas entre sí altitudinalmente, con 50 metros de diferencia, si bien suficientemente separadas en distancia (más de 700 metros) para considerarse dos complejos morrénicos diferenciados que señalan dos posiciones individuales del glaciar (tabla 1). Un nuevo y voluminoso complejo morrénico a $1424 \mathrm{~m}$ de altitud permite adscribir este a una nueva fase de avance glaciar (F-III). Finalmente una fase de glaciares de circo, de pequeñas dimensiones y con dos complejos, orientados al $\mathrm{E}$ y $\mathrm{N}$, señalan un nuevo equilibrio glaciar (F-IV). Este se acompaña de glaciares rocosos, dominantes como modelado frío en este periodo y en este circo, que señalan unas condiciones frías en altura, con presencia de permafrost de montaña.

En la vertiente septentrional los glaciares de Anzo y Monte Redondo no se han encontrado formas, depósitos ni complejos morrénicos que permitan la reconstrucción detallada de su evolución glaciar, más allá de una fase de máxima expansión y de las grandes formas erosivas.

En la vertiente meridional los cinco circos glaciares tienen una morfología neta y morrenas muy deterioradas, señalando el desarrollo de glaciares de circo alojados en altura con sus frentes entre 1670 y 1825 m s.n.m. y la ELA entre 1780 y 1880 metros de altitud, significativamente más altos que los de la vertiente norte. En los circos de Gildar Sur, Cosgaya, Los Verdes de Cable y La Requejada se pueden reconstruir los glaciares por las formas y algunos complejos morrénicos de la máxima expansión glaciar, pero no se aprecian fases de retroceso. Sólo los circos de Orza y Cebolleda SE presentan dos complejos morrénicos, muy claros en Orza, donde a una máxima expansión (F-I) le sigue un pequeño glaciar acantonado al pie de las cumbres de Montó (1996 m), por encima de los 1730 m s.n.m., de difícil adscripción morfocronológica. 
IMPLICACIONES CRONOLÓGICAS Y AMBIENTALES

La distancia y diferencia de altitud entre los complejos morrénicos cartografiados en cada valle y circo, así como la proximidad de la ELA de cada glaciar reconstruido y la localización y entidad de los mismos, que se presentan perfectamente individualizados, posibilita establecer la sucesión glaciomorfológica de cada valle y circo para con posterioridad establecer las fases de avance y equilibrio glaciar generalizables para toda la Sierra de Cebolleda. El establecimiento de estas fases de avance y retroceso glaciar permite su adscripción a fases cronológicas concretas por correlación con la evolución del glaciarismo en los macizos próximos donde se dispone de dataciones absolutas, y la reconstrucción de los cambios ambientales que condicionaron el glaciarismo de la Sierra.

\section{Fases y cronología glaciar}

La correlación morfológica y altitudinal de las formas de acumulación glaciar en la Sierra de Cebolleda permiten reconstruir la evolución glaciar durante el Pleistoceno, con cuatro fases de avance o equilibrio glaciar principales (Fig. 8), y pulsaciones menores en cada una de ellas.

- Fase I: Las morrenas externas señalan la máxima extensión de los glaciares, cuyos frentes quedaron de este modo alojados al norte en la conexión entre los valles laterales y el fondo de Valdeón. Publicaciones de divulgación recientes ${ }^{5}$ señalan una extensión de estos glaciares hacia la Garganta del Cares hasta alcanzar Puente Poncebos, 18 km más al norte, si bien no existen testigos morfológicos y sedimentarios glaciares que lo corroboren. En la vertiente sur se alojaron pequeños glaciares de circo con sus frentes 600 m más altos y las ELA medias también 330 m más arriba, mostrando una disimetría norte sur muy acusada. A su vez, también se aprecia un gradiente este oeste, pues al este los glaciares tuvieron un amplio desarrollo relativo, tanto al norte como al sur, bajo cumbres y crestas modestas que no superaban los 1850 metros, mientras al este no se desarrollaron bajo crestas de la misma altitud y superiores. Esta fase constituye el Máximo Glaciar Pleistoceno de la Sierra de Cebolleda, co-

5 Villa, E. (ed.)(2012): Parque Nacional de los Picos de Europa. Guía Geológica. IGME, OAPN, Madrid. 
rrelacionable en el estado actual de conocimientos con el máximo glaciar desarrollado en las montañas de su entorno inmediato (Picos de Europa, Fuentes Carrionas). Esta fase ha sido datada en 40-35 ka BP en el macizo Occidental de los Picos de Europa (Moreno et al. 2010) y anterior a 35,7-34,8 ka BP en el macizo Central (Serrano et al. 2012), acordes con otras dataciones realizadas en zonas más alejadas de la Cordillera Cantábrica (Jiménez y Farias, 2002; Jalut et al. 2010; Serrano et al. 2011; 2012; 2013), que sitúan la máxima extensión del hielo en el Estadio Isotópico Marino ${ }^{6} 3$, si bien recientes estudios en la Cordillera Cantábrica anticipan un máximo glaciar local en el MIS5/MIS4 (Frochoso et al. 2013).

FIGURA 8

EVOLUCIÓN GLACIAR DE LA SIERRA DE CEBOLLEDA

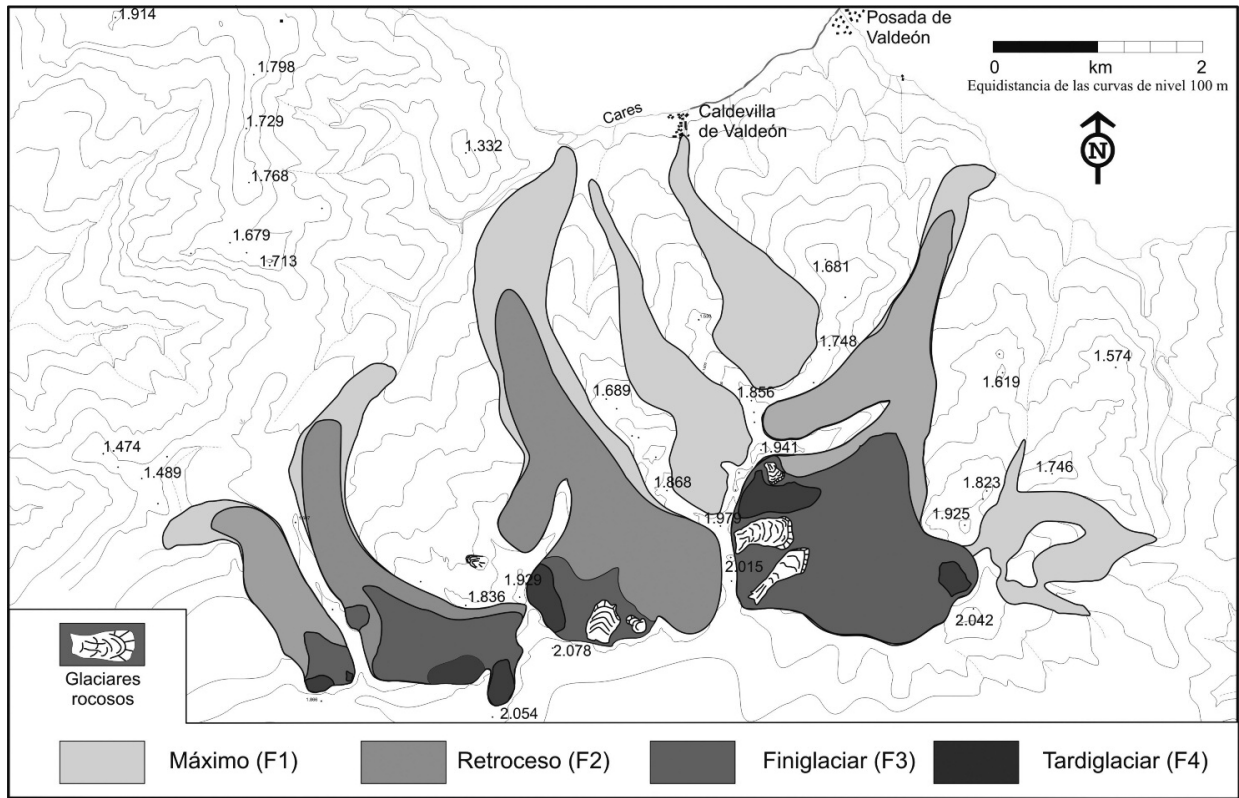

Fuente: elaboración propia.

${ }^{6}$ Estadio Isotópico marino, MIS en adelante.

Estudios Geográficos, Vol. LXXVI, 278, pp. 335-367, enero-junio 2015

ISSN: 0014-1496, eISSN: 1988-8546, doi: 10.3989/estgeogr.201512 
- Fase II: en las artesas glaciares y los circos las morrenas frontolaterales señalan una fase retroceso de glaciares con lenguas de $2-3 \mathrm{~km}$, al norte, o retranqueadas en nichos más altos, al sur. La proximidad de las morrenas con las de la fase anterior, no permite una neta diferenciación, si bien las morfologías son independientes, con arcos frontales y crestas laterales independientes que permiten atribuirlas a una fase de avance posterior al retroceso glaciar que sigue a la fase anterior. Las MELA se situaban 90 metros más altas de promedio que en la fase anterior, y los frentes $150 \mathrm{~m}$ más arriba en la vertiente norte. Estos hechos, junto al volumen de las morrenas, avalan la hipótesis de un avance o fase glaciar independiente de la fase I. En la sur, la ELA sólo se eleva $30 \mathrm{~m}$ y los frentes, sólo dos analizados, se situaban $15 \mathrm{~m}$ más arriba. En esta fase, en los glaciares orientados al sur no existen morrenas, por lo que se interpreta que desaparecen prácticamente, con sólo dos testigos, mientras al norte estaban bien desarrollados los glaciares en lengua. La disimetría de la fase anterior se hace más acusada, con una vertiente norte ampliamente glaciada y la sur sólo ocupada en las porciones más altas por pequeños glaciares y neveros. La proximidad altitudinal de las ELA y la proximidad de los frentes, que en Freñana, Cebolleda y Gabanceda forman parte de los mismos complejos morrénicos frontales, permiten interpretar que las fases I y II se localizan en periodos próximos en el tiempo, pertenecientes a una fase de máximo avance y un periodo de retroceso y equilibrio. Esta fase se correlaciona con el avance y estabilización registrado en las montañas de su entorno, si bien en este caso los frentes quedan más retranqueados que en los Picos de Europa o Fuentes Carrionas. En Áliva se ha establecido una cronología en torno a 24 ka BP para este periodo, acorde con las cronologías que para esta fase se han establecido en el valle del Trueba, posterior a 29,1-28,5 ka BP. Se trata de un importante periodo glaciar ubicado en el MIS2 y en relación con los periodos de máximo frío europeo, si bien en Cebolleda no significaría la máxima expansión glaciar.

- Fase III: en el interior de los valles, y sólo en la vertiente septentrional, los complejos morrénicos se localizan entre 1425 y $1590 \mathrm{~m}$ y en algunos casos, como en el circo de Freñana, muestran dos fluctuaciones muy próximas. Esta fase presenta la paleoMELA $100 \mathrm{~m}$ por encima de la anterior, y está bien representada en todos los circos de altura. Aunque la diferencia atitudinal de la ELA es pequeña entre las tres fases, los frentes quedan $250 \mathrm{~m}$ más altos y a gran distancia de los anteriores, $1,5 \mathrm{~km}$ en Frañana, 2,5 km en Cebolleda y $3 \mathrm{~km}$ en Gabanceda. Los glaciares eran 
de dimensiones más modestas, reduciendo su superficie más de un 70\% respecto a la fase I, con circos ampliamente glaciados pero lenguas muy cortas. La vertiente sur está completamente deglaciada. Se trata, pues de una fase con una fuerte disimetría norte-sur, con glaciares alojados a favor de orientación y altitud de los circos, con áreas de acumulación por encima de los 1600 m s.n.m. La altitud de la MELA, a 1700 m, ya indica que las condiciones derivadas del frío y la innivación favorecerían el desarrollo de los glaciares, frente a las condiciones topoclimáticas, como la sobrealimentación derivada del viento y la protección de las paredes septentrionales.

- Fase IV: las morrenas situadas en las porciones más altas de las laderas de los circos, siempre en orientaciones septentrionales, permiten reconstruir glaciares de circo de pequeñas dimensiones cuyos frentes se localizaban entre 1690 y 1860 m s.n.m., ubicados invariablemente bajo crestas con orientaciones de componente norte y este, excepto en Gabanceda, al NW. La paleoMELA de estos glaciares, estimada a $1785 \mathrm{~m}$ s.n.m., se ubicaba $85 \mathrm{~m}$ más alta que la anterior, en un periodo en el que la montaña estaba prácticamente deglaciada. Muy alejados de los frentes de la fase III, y 250 m más altos, son las condiciones topoclimáticas las

TABLA 2

GLACIARES ROCOSOS DE LA SIERRA DE CEBOLLEDA

\begin{tabular}{|c|c|c|c|c|c|c|c|c|}
\hline \multirow{2}{*}{ Nombre } & \multirow{2}{*}{ Coordenadas } & \multirow{2}{*}{$\begin{array}{c}\text { Altitud } \\
\text { Frente }\end{array}$} & \multirow{2}{*}{$\begin{array}{l}\text { (m) } \\
\text { Raíz }\end{array}$} & \multirow{2}{*}{$\begin{array}{c}\text { Long. } \\
\text { (m) }\end{array}$} & \multirow{2}{*}{$\begin{array}{c}\text { Anchura } \\
\text { (m) }\end{array}$} & \multirow{2}{*}{ Orient. } & \multirow{2}{*}{ Litología } & \multirow{2}{*}{ Fase } \\
\hline & & & & & & & & \\
\hline \multirow[t]{2}{*}{ Montó 1} & $43^{\circ} 6^{\prime} 14^{\prime \prime} \mathrm{N}$ & 1680 & 1800 & 505 & 162 & $\mathrm{NE}$ & Cuarcitas & IV \\
\hline & $4^{\circ} 55^{\prime} 27^{\prime \prime} \mathrm{W}$ & & & & & & & \\
\hline \multirow[t]{2}{*}{ Montó 2} & $43^{\circ} 634^{\prime \prime} \mathrm{N}$ & 1590 & 1850 & 750 & 300 & NE & Cuarcitas & IV \\
\hline & $4^{\circ} 55^{\prime} 29^{\prime \prime} \mathrm{W}$ & & & & & & & \\
\hline \multirow[t]{2}{*}{ Montó 3} & $43^{\circ} 650^{\prime \prime} \mathrm{N}$ & 1690 & 1740 & 135 & 90 & SE & Cuarcitas & IV \\
\hline & $4^{\circ} 55^{\prime} 20^{\prime \prime} \mathrm{W}$ & & & & & & & \\
\hline \multirow[t]{2}{*}{ Horcada Cable } & $43^{\circ} 59^{\prime \prime} \mathrm{N}$ & 1690 & 1890 & 650 & 420 & $\mathrm{~N}$ & Cuarcitas & IV \\
\hline & $4^{\circ} 56^{\prime} 16^{\prime \prime} \mathrm{W}$ & & & & & & & \\
\hline \multirow[t]{2}{*}{ Verdes de Cable } & $43^{\circ} 69^{\prime \prime} \mathrm{N}$ & 1820 & 1920 & 290 & 156 & NW & Cuarcitas & IV \\
\hline & $4^{\circ} 56^{\prime} 35^{\prime \prime} \mathrm{W}$ & & & & & & & \\
\hline
\end{tabular}

Fuente: elaboración propia. 
que condicionan los emplazamientos. Los glaciares se alojaban en umbrías con paredes verticales (Gabanceda, Frañana), o con componentes este, sobrealimentadas por los vientos del W y NW. En este periodo se desarrollaron en las cabeceras de Frañana, Gildar y Gabanceda, 5 glaciares rocosos de longitudes entre 135 y $750 \mathrm{~m}$, ubicados por encima de los 1590 m s.n.m. y todos ellos en orientación norte (tabla 2). Su presencia indica condiciones ambientales con permafrost por encima de $1830 \mathrm{~m}$ s.n.m. Es, pues, una fase fría, donde la persistencia o sobreacumulación de la nieve permiten el desarrollo de pequeños glaciares en altitud.

Las formas pertenecientes a la fases III y IV se correlacionan con fases glaciares de las montañas más próximas que han sido adscritas al Tardiglaciar en Picos de Europa (Serrano et al. 2002; González Trueba, 2007; Pellitero et al. 2011; Serrano et al. 2012). En Áliva se ha establecido un avance glaciar, correlacionado con la glaciación de los circos en la alta montaña, en torno a 14 ka. BP (Serrano et al. 2012) y en el lago de Enol se ha establecido un periodo frío entre 14,5 y 13,5 ka BP (Moreno et al. 2010), de modo que los glaciares de altitud y la generación de glaciares rocosos en los circos de la Sierra de Cebolleda se pueden correlacionar con las fases frías registradas en Europa durante el Dryas reciente (14-12 ka BP).

\section{Cambios ambientales del glaciarismo en Sierra Cebolleda}

La proximidad de los frentes y de las ELA en las fases I y II (15 m de diferencia altitudinal) señala unas condiciones similares y la posibilidad de que se enmarquen en un solo ciclo glaciar (tabla 3). Los glaciares estaban alimentados por abundantes precipitaciones, con acceso del N y del NW y la orientación fue determinante, dada la fuerte disimetría glaciar del macizo. No existen huellas de frío intenso ajenas a los glaciares en las laderas meridionales, donde los glaciares ocupaban muy poca extensión, por lo que el frío estaría acompañado de intensas precipitaciones nivales. Para ello, y dada la protección del macizo por el norte, donde se encuentran los Picos de Europa, una explicación a la intensa innivación sería la llegada de precipitaciones nivales del NW y del SW que propiciarían el amplio desarrollo de los glaciares. Las huellas morfológicas de la Sierra de Cebolleda no permite, pues, diferenciar condiciones ambientales para estas fases, si bien, atendiendo al conjunto de la Cordillera Cantábrica central (Serrano et al. 2013) podría corresponder a un comportamiento diferenciado entre ambas fases. La primera ha sido definida 
TABLA 3

SÍNTESIS DE LAS ELA Y ALTITUDES MEDIAS DE LOS FRENTES

EN CADA FASE GLACIAR

\begin{tabular}{ccccccc}
\hline & \multicolumn{3}{c}{ ELA m s.n.m. } & \multicolumn{3}{c}{ Altitud de los frentes m s.n.m. } \\
\cline { 2 - 7 } Fases & \multicolumn{3}{c}{ Orientación } & & \multicolumn{3}{c}{ Orientación } \\
\cline { 2 - 7 } & N & S & X & N & S & X \\
\cline { 2 - 7 } I & 1500 & 1840 & 1655 & 1120 & 1715 & 1390 \\
II & 1595 & 1810 & 1640 & 1275 & 1730 & 1645 \\
III & 1700 & & 1700 & 1516 & & 1516 \\
IV & 1785 & & 1785 & 1729 & & 1729 \\
\hline
\end{tabular}

Fuente: elaboración propia.

como una expansión a favor de condiciones muy húmedas con elevada innivación procedente del SW. En la segunda fase, separada 20.000 años de la anterior, las condiciones frías del LGM europeo posibilitaron que los glaciares, peor alimentados pero mejor conservados, alcanzasen cotas similares a la fase anterior (Serrano et al. 2012, 2013). Ambas responden, en cualquier caso, a unas intensas precipitaciones nivales y escasa fusión estival, bien por sobrealimentación nival, en el primer caso, bien por condiciones de frío intenso en el segundo.

A partir de estas fases glaciares las ELA se alejan más en cada periodo de avance glaciar, 65 metros entre las fases II y III, y 85 entre las III y IV. Durante las fases III y IV los glaciares son cortos y se alojan en altitud, incapaces de desarrollar lenguas ni avanzar en exposiciones sur. Pero mientras la fase III ocupa todos los circos del norte, la fase IV sólo están ocupadas por glaciares las laderas con condiciones topoclimáticas adecuadas. La escasez de huellas periglaciares correlacionables con la fase III denota unas moderadas precipitaciones y un incremento del frío poco acusado. Los glaciares avanzan al norte, pero no se generan en las orientaciones meridionales, por lo que se interpreta como un periodo de equilibrio en el retroceso relacionado con un enfriamiento menor, con temperaturas estivales más moderadas. Este hecho implicaría el estacionamiento y avance de los glaciares durante el retroceso del LGM, una vez desaparecidos en la vertiente sur. 
La ELA de la fase IV se aloja ya muy separada de las anteriores pero sobre todo los glaciares se emplazan únicamente en las caras norte de los circos, en ambientes topoclimáticos favorables. Allí donde no se desarrollan glaciares existen glaciares rocosos, indicadores de frío intenso y ambientes con permafrost. Las formas glaciares y periglaciares denotan unas condiciones de moderadas precipitaciones pero frío intenso con escasa fusión estival allí donde no hay incidencia de la radiación solar. Un enfriamiento de las condiciones estivales y pocas aportaciones nivales permiten explicar un ambiente frío, pero menos que en las fases I y II, y relativamente seco, con veranos frescos que propician la conservación de cuerpos helados por debajo de los 1600 m s.n.m. Los cinco glaciares rocosos existentes (tabla 2) emplazaron sus frentes entre 1590 y 1820 m s.n.m. en orientaciones N (85\%), NE y NW, y son del mismo periodo. El emplazamiento de los frentes de los glaciares rocosos necesita temperaturas medias máxima menores de $-2^{\circ} \mathrm{C}$, mientras las raíces se asocian a la isoterma de $-6^{\circ} \mathrm{C}$. De este modo, en torno a los $1700 \mathrm{~m}$ s.n.m. la temperatura media anual del aire (TMAA) sería al menos de $-2 /-6^{\circ} \mathrm{C}$. Manteniendo el gradiente altitudinal de la montaña, establecido en $0,52-0,54^{\circ} \mathrm{C} / 100 \mathrm{~m}$ (Allende, 2008), podemos estimar que a $1000 \mathrm{~m}$ la TMAA en este periodo sería aprox. $+1,7 /-2,2^{\circ} \mathrm{C}$.

En la actualidad las estaciones más próximas, en el Esla (Riaño) y Sajambre (Soto de Sajambre) (tabla 4) tienen condiciones húmedas e hiperhúmedas a favor de la llegada de masas húmedas del N, NW y SW (Rivas et al. 1984; Allende, 2008), con unas temperaturas medias anuales de $8,4^{\circ} \mathrm{C}$ y $9,3^{\circ} \mathrm{C}$. Aplicando el mismo gradiente altitudinal de las montañas templadas, a $1700 \mathrm{~m}$ la TMAA sería aprox. $5,4-5,1^{\circ} \mathrm{C}$.

TABLA 4

CONDICIONES CLIMÁTICAS ACTUALES Y ESTIMACIONES PARA LA FASE IV.

\begin{tabular}{ccccccc}
\hline Estación & $\begin{array}{c}\text { Altitud } \\
\text { m s.n.m. }\end{array}$ & $\begin{array}{c}\text { Precipitación } \\
\mathrm{mm} \mathrm{a}^{-1}\end{array}$ & $\mathrm{~T}^{\mathrm{a}} \mathrm{MA}{ }^{\circ} \mathrm{C}$ & Clima & $\begin{array}{c}\text { Estimación T } \\
\text { a } 1700 \mathrm{~m}\end{array}$ & $\begin{array}{c}\text { Estimación T } \\
1700 \mathrm{~m} / \mathrm{F}-\mathrm{III}\end{array}$ \\
\hline $\begin{array}{c}\text { Soto de } \\
\text { Sajambre }\end{array}$ & 900 & 1700 & 9,3 & $\begin{array}{c}\text { Hiper } \\
\text { húmedo }\end{array}$ & $5,4^{\circ} \mathrm{C}$ & $-2,6^{\circ} \mathrm{C}$ \\
Riaño & 1048 & 1200 & 8,4 & $\begin{array}{c}\text { Montano } \\
\text { húmedo }\end{array}$ & $5,1^{\circ} \mathrm{C}$ & \\
\hline
\end{tabular}

Fuente: elaboración propia. 
De este modo, la diferencia térmica entre las TMAA actual y de la fase IV sería aprox. de $6,8 / 7,6^{\circ} \mathrm{C}$. Si el gradiente térmico es más bajo, como es propio de montañas hiperhúmedas, esta diferencia puede ser menor. Dadas las temperaturas estimadas para el LGM en Europa, con un descenso térmico de $8^{\circ} \mathrm{C}$ (Allen et al. 2008) y las estimadas en Escocia para el Tardiglaciar (Benn y Ballantyne, 2005), de $4^{\circ} \mathrm{C}$ para las TMA de verano (siempre más moderadas que las medias anuales) y con precipitaciones en torno a $1970 \mathrm{~mm} \mathrm{a}^{-1}$, el rango de temperaturas estimado para este periodo podría estar acorde, aunque refleja un frío más intenso, con lo sucedido en los glaciares europeos durante la última fase de avance glaciar Pleistoceno. Un marcado descenso de las precipitaciones y una temperaturas frías, aprox. $7^{\circ} \mathrm{C}$ más bajas que las actuales como máximo, posibilitaron el desarrollo de pequeños glaciares en los circos orientados al norte y por encima de los $1860 \mathrm{~m}$, y ambientes con permafrost en las orientaciones menos favorables hasta los $1590 \mathrm{~m}$, con génesis de glaciares rocosos y un dominio periglaciar de alta montaña.

\section{CONCLUSIONES}

Las montañas de la divisoria cantábrica, protegida por los más altos macizos al $\mathrm{N}$ y al S, presentan un comportamiento diferenciado frente a estos últimos, que se concreta en una fuerte disimetría N-S de los procesos y formas asociados al frío, y en particular el glaciarismo cuaternario. La morfología de la Sierra de Cebolleda se caracteriza por su englaciamiento durante distintos periodos del Cuaternario, a pesar de la baja cota de cumbres y cordales (19002040 m s.n.m.). Durante el máximo glaciar Pleistoceno la MELA se situó en torno a 1500 m s.n.m. al N, y a 1600 m s.n.m. al S. Estas son las cotas del umbral límite de glaciación en las montañas centrales de la divisoria, donde la altitud fue el factor limitante para el desarrollo del máximo glaciar.

En la Sierra de Cebolleda se aprecian dos fases glaciares de extensiones similares y próximas en el tiempo, la de máxima expansión (F.1) y un retroceso y equilibrio (F.2), correlacionables con lo sucedido en los macizos del entorno (Picos de Europa, Peña Prieta-Curavacas). En ambos periodos las precipitaciones nivales, moderadas por el efecto pantalla de los grandes macizos situados al norte y al sur, propiciaron el desarrollo del glaciarismo, que se concreta en un largo periodo de equilibrio tras una máxima expansión de menor entidad.

Las morrenas intermedias, localizadas en el interior de los valles, se relacionan con un periodo de retroceso y equilibrio Finiglaciar (F.3), con comportamientos diferenciados para cada glaciar, condicionados por los factores 
topoclimáticos locales y las condiciones de emplazamiento (desnivel, topografía previa, litología). Este hecho se concreta en la ubicación de la MELA, al N, a 1700 m s.n.m., a tan sólo 200 metros de las cumbres y ya en el límite de la glaciación, lo que conlleva la diferenciación entre valles de los restos morfológicos.

En altitud, ya en el interior de los circos, los restos glaciares (F.4) denotan un periodo glaciar marginal, caracterizado por pequeños glaciares desarrollados a favor de las condiciones topoclimáticas. Glaciares de circo, sin lenguas, que comparten las cabeceras de los valles con glaciares rocosos. Estos últimos denotan la presencia de permafrost por encima de 1600 m s.n.m., 200 metros por debajo de la MELA en esta fase. Se trata por tanto un periodo de frío intenso en la alta montaña, con temperaturas medias anuales al menos inferiores a $-2^{\circ} \mathrm{C}$, y con una innivación moderada, derivada de unas condiciones climáticas secas favorecidas por la protección ejercida por los macizos circundantes frente a los vientos del N y NW.

Ambos factores generaron unas condiciones climáticas secas y frías en la alta montaña de la divisoria, capaces de originar intensos procesos periglaciares, tales como glaciares rocosos, laderas de bloques, corrientes de bloques, derrubios ordenados, pero un moderado desarrollo del glaciarismo. Por correlación, y a falta de dataciones para el conjunto de la Cordillera Cantábrica, se sitúa temporalmente en la última fase fría cuaternaria, el Tardiglaciar.

El estudio detallado de la evolución glaciar de los diferentes macizos de la Cordillera Cantábrica y la datación de las distintas fases, desde el Máximo Glaciar hasta el Holoceno, son cruciales para el conocimiento de las fases frías del Pleistoceno, la evolución del clima y la reconstrucción de ambientes naturales entre MIS3 y MIS1, cuando el poblamiento humano moderno inicia la ocupación de estas montañas.

Fecha de recepción: 9 de mayo de 2013.

Fecha de aceptación: 17 de septiembre de 2013.

\section{BiBLIOGRAFÍA}

Allen, R.; Siegert, M. J. y Payne, A. J. (2008): "Reconstructing glacier-based climates of LGM Europe and Russia-Part 2: A dataset of LGM precipitation/ temperature relations derived from degree-day modelling of palaeo glaciers". Climate of the Past, 4, pp. 249-263.

Allende, F. (2008): "Una aproximación a la caracterización climática de un sector de la montaña central cantábrica". Estudios Geográficos, 265, pp. 357-383. 
Alonso Herrero, E. (1987): "Huellas del glaciarismo cuaternario en las cabeceras del río Esla, vertiente sur de la Cordillera Cantábrica (León)". Cuaternario y Geomorfología, 1, pp. 49-59.

Alonso Herrero, E. (2002): "El glaciarismo en las cuencas altas de los ríos Esla y Porma”, en J. M. Redondo, A. Gómez, R. B. González y P. Carrera (eds.): El modelado de origen glaciar en las montañas leonesas. León, Universidad de León, pp. 235-248.

Alonso, F; Arenillas, M. y Saenz, C. (1982): "La morfología glaciar en las Montañas de Castilla La Vieja y León", en I Congreso de Geografía de Castilla-León, Burgos, Concejo General de Castilla y León, pp. 23-41.

Bakke, J. y Nesje, A (2011): "Equilibrium line altitude (ELA)", en V. P. Singh, P. Singh y U. K. Haritashya (eds.). Encyclopaedia of Snow, Ice and Glaciers. Heidelberg, Springer, pp. 979-984.

Ballantyne, C. K. (2002): "The Loch lommond Advance on the Isle of Mull, Scotland: glacier reconstruction and palaeoclimatic implications". Journal of Quaternary Science, 17, pp. 759-771.

Barsch, D. (1996): Rock Glaciers. Indicators for the Present and Former Geoecology in High Mountain Environment. Heidelberg, Springer Verlag.

Benn, D. I. y Ballantyne, C. K. (2005): "Paleoclimatic reconstruction from Loch Lommond readvance glaciers in the west Drumochter Hills, Scotland". Journal of Quaternary Research, 20, pp. 577-592.

Brouwer, A. (1964): "Deux facies dans le Devonien des Montagnes Cantabriques meridionales". Boletín del Instituto de Estudios Asturianos, 10, pp. 1-10.

Castañón, J. C. (1989): "Las formas de relieve de origen glaciar en los sectores central y oriental del macizo asturiano". Tesis doctoral, Universidad de Oviedo.

Castañón, J. C. y Frochoso, M. (1992): "La glaciación Würm en las montañas cantábricas", en C. Cearreta y F. Ugarte (eds.): The late Quaternary in the Western Pyrenean Region. Bilbao, Universidad del País Vasco, pp. 319-332.

Dahl, S. O. y Nesje, A. (1992): "Paleoclimatic implications based on equilibrium-line altitude depressions of reconstructed Younger Dryas and Holocene cirque glaciers in inner Nordfjord, western Norway". Palaeogeography, Palaeoclimatology, Palaeoecology, 94, pp. 87-97.

Frankenfeld, H. (1983): "El manto de Montó-Arauz: interpretación estructural de la Región del Pisuerga-Carrión (Zona Cantábrica, NW de España)". Trabajos de Geología, 13, pp. 37-47.

Frochoso, M. y Castañón, J. C. (1998): "El relieve glaciar de la Cordillera Cantábrica", en A. Gómez Ortiz y A. Pérez Alberti (eds.): Las huellas glaciares de las montañas españolas. Santiago de Compostela, Servicio de Publicaciones de la Universidad de Santiago de Compostela, pp. 65-137.

Frochoso, M.; González, R. y Allende, F. (2013): "Pleistocene glacial morphology and timing of last Glacial cycle in Cantabrian Mountains (Northern Spain): new chronological data from the the Asón area". Central European Journal of Geosciences, 5/1, pp. 12-27. 
González Gutiérrez, R. B. (2002): El relieve de los valles del Torío y Curueño (Montaña Cantábrica Leonesa). León, Universidad de León.

González Trueba, J. J. (2006): "Topoclimatical factors and very small glaciers in Atlantic Mountain of SW Europe: Little Ice Age glacier advance in Picos de Europa (NW Spain)". Zeitschrift für Gletscherkunde und Glazialgeologie, 39, pp. 115-125.

González Trueba, J. J. (2007a): El paisaje natural del Macizo Central de los Picos de Europa. Santander, Centro de Investigación del Medio Ambiente (CIMA), Consejería de Medio Ambiente, Gobierno de Cantabria.

González Trueba, J. J. (2007b): Geomorfología del Macizo Central del Parque Nacional Picos de Europa. Madrid, OAPN/Ministerio de Medio Ambiente.

González Trueba, J. J. (2007c): La Pequeña Edad del Hielo en los Picos de Europa. Análisis y reconstrucción del avance glaciar histórico (s.XIX). Santander, Universidad de Cantabria/Fundación Marcelino Botín.

González Trueba, J. J., Moreno, R., Martínez de Pisón, E. y Serrano, E. (2008): "Little Ice Age glaciation and current glaciers in the Iberian Peninsula". The Holocene, 18, pp. 569-586.

González Trueba, J. J. y Serrano, E. (2010): Geomorfología del Macizo Oriental del Parque Nacional Picos de Europa. Madrid, OAPN/Ministerio de Medio Ambiente.

González Trueba, J. J., Serrano, E., González García, M., Gómez Lende, M. y Ruiz, J. (2011): Geomorfología del Macizo Occidental del Parque Nacional Picos de Europa. Madrid, OAPN-Ministerio de Medio Ambiente.

González Trueba, J. J., Gómez Lende, M., González García, M. y Serrano, E. (2012): "Mapa Geomorfológico de la Orla Sur del Parque Nacional de los Picos de Europa. OAPN-Ministerio de Medio Ambiente", Madrid, Gobierno de España (inédito).

Haeberli, W.; Hallet, B.; Arenson, L.; Elconin, R.; Humlum, O.; Kääb, A.; Kaufmann, V.; Ladanyi, B.; Matsuoka, N.; Springman, S. y Vonder Mühl, D. (2006): "Permafrost creep and rock glacier dynamics". Permafrost and Periglacial Proceses, 17 , pp. 189-214.

Hawkins, F. J. (1985): "Equilibrium Line altitude and paleoenvironment in the Merchant Bay area, Baffin island, N.W.T., Canada". Journal of Glaciology, 31 (109), pp. 205-213.

Heredia, N. y Rodríguez Fernández, L. R. (1990): Memoria Mapa Geológico de España E. 1:50.000 no 80. Burón. Madrid, ITGE.

Hughes, P. D. (2010): "Geomorphology and quaternary stratigraphy: the roles of morpho-, litho-, and allostratigraphy". Geomorphology, 123, pp. 189-199.

Jalut, G.; Turu, V.; Dedoubat J. J.; Otto, T.; Ezquerra, J.; Fontugne, M.; Belet, J. M.; Bonnet, J.; García, A.; Redondo, J. M.; Vidal, J. R. y Santos, L. (2010): "Palaeoenvironmental studies in NW Iberia (Cantabrian range): Vegetation history and synthetic approach of the last deglaciation phases in the western Mediterranean". Palaeogeography, Palaeoclimatology, Palaeoecology, 297, pp. 330-350.

Jiménez, M.; Rodríguez-Rodríguez, L.; García-Ruiz, J. M.; Domínguez-Cuesta, M. J.; Farias, P.; Valero-Garcés, B.; Moreno, A.; Rico, M. y Valcárcel, M. (2012): “A review 
of glacial geomorphology and chronology in northern Spain: Timing and regional variability during the last glacial cycle". Geomorphology, 196, pp. 50-64.

Jiménez, M. y Farias, P. (2002): "New radiometric and geomorphologic evidences of a last glacial maximum older than $18 \mathrm{ka}$ in SW European Mountains: the example of Redes Natural Park (Cantabrian Mountains, NW Spain)". Geodinamica Acta, 15, pp. 93-101.

Julivert (1971): "Decollement tectonics in the Hercynian Cordillera of NW Spain". American Journal of Science, 270, pp. 1-29.

Kuhle, M. (1988): "Topography as a fundamental element of glacial systems. A new approach to ELA calculation and typological classification of paleo-and recent glaciations". GeoJournal, 17/4, pp. 545-568.

Lukas, S. (2006): "Morphostratigraphic principles in glacier reconstruction -a perspective from the British Younger Dryas". Progress in Physical Geography, 30, pp. 719-736.

Marquínez, J. y Marcos, A. (1984): "La estructura de la unidad del Gildar-Montó (Cordillera Cantábrica)". Trabajos de Geología, 14, pp. 53-64.

Moreno, A.; Valero Garcés, B. L.; Jiménez, M.; Domínguez, M. J.; Mata, M. P.; Navas, A.; González Sampériz, P.; Stoll, H.; Farias, P.; Morellón, M.; Corella, J. P. y Rico, M. T. (2010): "The last glaciation in the Picos de Europa Nacional Park (Cantabrian Mountains, northern Spain)". Journal of Quaternay Science, 25, pp. 10761091.

Nesje, A. (2006): "Paleo ELAs", en S. A. Elias (ed.): Encyclopaedia of Quaternary Science. Amsterdam, Elsevier, pp. 882-892.

Ohmura, A.; Kasser, P. y Funk, M. (1992): "Climate at the equilibrium line of glaciers". Journal of Glaciology, 38, pp. 397-411.

Pellitero, R. (2009): "Application of an alpine geomorphological mapping system to an atlantic mountain environment: The Curavacas Massif (Cantabrian Range, Northwest Spain)". Journal of Maps, 5/1, pp. 194-205.

Pellitero, R. (2012): "Geomorfología, paleoambiente cuaternario y geodiversidad en el macizo de Fuentes Carrionas - Montaña Palentina. Tesis doctoral, Universidad de Valladolid.

Pellitero, R. (2013): "Evolución finicuaternaria del glaciarismo en el macizo de Fuentes Carrionas (Cordillera Cantábrica), propuesta cronológica y paleoambiental". Cuaternario y Geomorfología, 27/1-2, pp.71-90.

Pellitero, R., Serrano, E. y González Trueba, J. J. (2011): "Glaciares rocosos del sector Central de la Montaña Cantábrica: indicadores paleoambientales". Cuadernos de Investigación Geográfica, 37/29, pp. 119-144.

Pérez-Alberti, A., Valcárcel, M. y Blanco, R.(2004): "Pleistocene glaciation in Spain", en J., Ehlers y P. L., Gibbard (eds.): Quaternary Glaciations-Extent and Chronology. Elsevier, London, pp. 389-394.

Porter, S. C. (1975): "Equilibrium line altitudes of Late Quaternary glaciers in the Southern Alps, New Zealand". Quaternary Research, 5, pp. 27-47. 
Redondo, J. M.; Gómez, A.; González, R. B. y Carrera, P. (eds.) (2002): El modelado de origen glaciar en las montañas leonesas. León. Universidad de León.

Rivas Martínez, S.; Díaz, T. E.; Prieto, J. A. F; Loidi, J. y Penas, A. (1984): Los Picos de Europa. La vegetación de la alta montaña cantábrica. León, Ediciones leonesas.

Rodríguez Fernández, L. R. y Heredia, N. (1988): "La estratigrafía del Carbonífero y la estructura de la unidad del Pisuerga-Carrión. NO de España”. Cadernos Laboratorio Xeologico de Laxe, 12, pp. 207-229.

Santos, J. (2011): "Glaciarismo y periglaciarismo en el Alto Sil, provincia de León (Cordillera Cantábrica)". Tesis doctoral, Universidad de León.

Seltzer, G. O. (1994): "Climatic interpretation of alpine snowline variations on millennial time scales". Quaternary Research, 41, pp. 154-159.

Serrano, E. y González Trueba, J. J. (2002): "Morfología y evolución glaciar en los Picos de Europa”, en J. M. Redondo, A. Gómez, R. S. González y P. Carrera (eds.): El modelado de origen glaciar en las montañas leonesas. León, Servicio de Publicaciones de la Universidad de León, pp. 249-268.

Serrano, E.; González Trueba, J. J.; Turu, V. y Ros, X. (2011): "Cronología glaciar pleistocena en el valle de Trueba (Cordillera Cantábrica): primeras dataciones", en V. Turu y A. Constante (eds.): El Cuaternario en España y áreas afines: avances en 2011. Andorra, AEQUA-Fundación M. Chevalier, pp. 3-6.

Serrano, E.; González Trueba, J. J. y González García, M. (2012): "Mountain Glaciation and Paleoclimate Reconstruction in the Picos de Europa (Iberian Peninsula, SW Europe)". Quaternary Research, 78, pp. 303-314.

Serrano, E.; Gómez Lende, M.; González Trueba, J. J.; Turu, V. y Ros, X. (2013): "Fluctuaciones glaciares pleistocenas y cronología en las Montañas Pasiegas (Cordillera Cantábrica)". Cuaternario y Geomorfología, 27/1-2, pp.91-110.

Serrano, E.; González-Trueba, J. J.; Pellitero, R.; González-García, M. y Gómez-Lende, M. (2013): "Quaternary glacial evolution in the Central Cantabrian Mountains (Northern Spain)”. Geomorphology, 196, pp. 65-82.

\section{RESUMEN}

La Sierra de Cebolleda constituye $10 \mathrm{~km}$ de la divisoria cantábrica, en dirección E-W, al sur de los Picos de Europa, el macizo más alto de la cordillera. Su elevada diversidad litológica y la estructura monoclinal condicionan el glaciarismo cuaternario. Este trabajo estudia la evolución glaciar mediante las huellas geomorfológicas. El objetivo es conocer la evolución y extensión temporal del glaciarismo para establecer correlaciones lo más precisas posibles con los restantes macizos cantábricos. Se ha realizado una cartografía geomorfológica E.1/25.000, la estimación de la ELA en cada glaciar y para cada fase temporal y un análisis morfoestratigráfico incluyendo datos sobre la orientación, la altura de los frentes, la longitud de los glaciares y la ELA. Se han estudiado y reconstruido 11 sistemas glaciares, con lenguas de más de $5 \mathrm{~km}$ que alcanzan en la vertiente norte una altitud media de 1120 m s.n.m. Los complejos morrénicos 
señalan entre una y cuatro fluctuaciones glaciares que permiten establecer cuatro fases glaciares: dos de extensión similar (F1 y F2), un periodo de retroceso y equilibrio Finiglaciar (F3), y un periodo glaciar marginal con pequeños glaciares en el interior de los circos (F4) e intensos procesos periglaciares (glaciares rocosos, laderas de bloques, corrientes de bloques, derrubios ordenados). Finalmente, estas fases se correlacionan con las dataciones existentes en otros macizos de la Cordillera Cantábrica. a Prieta-Curavacas).

PALAbras Clave: geomorfología; glaciarismo; Glaciaciones Cuaternarias; Cordillera Cantábrica.

\section{AbSTRACT}

The Sierra de Cebolleda (León province, Castilla y León) is a $10 \mathrm{~km}$ length part of tha Cantabrian Divide, located to the South of Picos de Europa, the highest massif in the Cantabrian Mountain. The high lithological diversity and monocline structure have addressed the Quaternay glaciation. The paper studies the glacial evolution by mean of the glacial features and the objective is to know the temporal evolution and extent of Quaternary glaciers and to establish as accurate as possible correlations with the other Cantabrian massifs. A geomorphological map to scale 1/25,000, ELA calculation of each glacier and glacial period, and a morphostrtigraphical analysis, including aspect, front altitudes, glaciers lenght and ELA have been made. Eleven glaciers systems have been reconstructed, defining in the North side tongues longer than $5 \mathrm{~km}$ and a medium altitude of fronts of $1120 \mathrm{~m}$ a.s.l. The moraine complex point to between one and four glacial stages in each valley. Four glacial stages have been establish: two of alike extent (F1 and F2), a retreated and equilibrium stage (F3) and a marginal glacial stage defined by small cirque glacier (F4) and extreme periglacial processes (rock glaciers, block slopes, block streams and stratified debris). Finally, the glacial stages have been correlated with known absolute dating of other massif of the Cantabrian Mountain.

KEY WORDS: geomorphology; glaciers landforms; Quaternary glaciations; Cantabrian Mountains.

\section{RÉSUMÉ}

La Sierra de Cebolleda fait parte de la tournant Cantabrique au $10 \mathrm{~km}$ de longueur, du direction E-W, au sud de la Picos de Europa, le plus haut massif de la Cordillère. Sa haute diversité lithologique et la structure monoclinique détermine la glaciation Quaternaire. Cet article étudie l'évolution géomorphologique glaciaire pour ses empreintes géomorphologiques. Lobjectif est de comprendre l'évolution et l'extension temporelle de la glaciation pour d'établir des corrélations les plus précises possibles avec l'autres massifs Cantabriques. Il ya eu une cartographie géomorphologique $\mathrm{E}$ $1 / 25.000$, une estimation de la ELA dans chaque glacier et chaque phase et une analyse morphostratigraphique, y compris des données sur l'orientation, la hauteur des 
fronts, la longueur des glaciers et la ELA. Ont été étudiés et reconstruit 11 glaciers, avec langues plus de 5 kilomètres, atteindre dans le versant nord une altitude moyenne de $1120 \mathrm{~m}$ d'altitude. Les moraines permettre à l'établissement de quatre glaciers phases. Deux du prolongation similaire (F1 et F2), une période de déclin et d'équilibre glaciaire (F3) et une période glaciaire marginal de petits glaciers du cirque (F4) avec processus périglaciaires intenses (glaciers rocheux, pentes de blocs, courants de blocs, éboulis ordonnés). Enfin, ces phases sont en corrélation avec les datations existants dans d'autres massifs des Monts Cantabriques.

Mots CLÉs: géomorphologie; formes glaciaires; glaciations quaternaires; Cordillère Cantabrique. 